\title{
Methylenphosphanyl-Komplexe von Chrom, Molybdän, Wolfram, Eisen und Nickel: Synthese und Reaktionen ${ }^{\text {मे }}$
}

\author{
Edgar Niecke*a , Hans-Jürgen Metternich ${ }^{\mathrm{a}}$, Martin Nieger ${ }^{\mathrm{a}}$, Dietrich Gudat ${ }^{\mathrm{a}}$, Peter Wenderoth ${ }^{\mathrm{a}}$, Wolfgang Malisch*b, \\ Christoph Hahner ${ }^{\mathrm{b}}$ und Wolfgang Reich ${ }^{\mathrm{b}}$
}

Anorganisch-Chemisches Institut der Universität Bonn ${ }^{\mathrm{a}}$,

Gerhard-Domagk-Straße 1, W-5300 Bonn 1

Anorganisch-Chemisches Institut der Universität Würzburg ${ }^{\mathrm{b}}$,

Am Hubland, W-8700 Würzburg

Eingegangen am 16. Oktober 1992

Key Words: Methylenephosphanyl complexes / Phosphenium complexes / Phosphavinylidene complexes

\begin{abstract}
Methylenephosphanyl Complexes of Chromium, Molybdenum, Tungsten, Iron and Nickel - Synthesis and Reactivity ${ }^{2}$
The reaction of the functionalized $\mathrm{P} / \mathrm{C}$ double bond systems $\mathrm{X}-\mathrm{P}=\mathrm{C}(\mathrm{R}) \mathrm{SiMe}_{3}\left[\mathrm{R}=\mathrm{SiMe}_{3}\right.$ and $\mathrm{X}=\mathrm{Cl}(\mathbf{1} \mathrm{a}), \mathrm{Cp}^{*}(\mathbf{5} \mathrm{a})$ or $\mathrm{R}$ $=\mathrm{Ph}$ and $\mathrm{X}=\mathrm{Br}(\mathbf{1} \mathbf{b}), \mathrm{Cp} *(\mathbf{5} \mathbf{b})]$ with alkali carbonylmetallates or carbonyl complexes of group 6, 8, and 10 metals leads to the formation of the transition metal-substituted phosphaalkenes $\left[\{M\}-P=C(R) S_{i M e} \mathbf{3 a}-\mathbf{j}\right.$ and $\left.\mathbf{4} \mathbf{a}-\mathbf{i}\right]$, in which the $\mathrm{P}-\mathrm{C}$ fragment acts as a one-electron donor. Depending on the nucleophilicity of the transition metal anion used, but also on the methylene carbon substituents [1 a: $\left(\mathrm{SiMe}_{3}\right)_{2} ; \mathbf{1 b}$ : $(\mathrm{Ph})-$ $\mathrm{SiMe}_{3}$ ), a different stability of the (methylenephosphanyl)-

trum of reactivities. As demonstrated by the reactions of the (methylenephosphanyl)metal complexes with sulfonic acid derivatives $(10 \mathbf{a}-\mathbf{c})$, diverse transition metal complexes $(\mathbf{1 1} \mathbf{a}-\mathbf{c})$, as well as chalcogenes $(\mathbf{1 4} \mathbf{a}-\mathbf{c}, \mathbf{1 5})$, a change in the reactivity is observed, indicating a transition from an olefinic to a carbenic character of the methylenephosphane. In comparison to the phenyl-substituted derivatives $4 a-i$ the bis(trimethyl)substituted species $\mathbf{3 a}-\mathbf{j}$ exhibit an increased reactivity. This is reflected in the easy decarbonylation of $\mathbf{3 a} \mathbf{a}-\mathbf{d}$ to the phosphavinylidene complexes $12 \mathrm{a}-\mathbf{d}$.
\end{abstract} metal complexes is observed, which results in a broad spec-
Übergangsmetallsubstituierte Methylenphosphanderivate, in denen das P/C-Doppelbindungssystem als Einelektronendonor gegenüber einem Übergangsmetallatom fungiert, werden seit einigen Jahren intensiv erforscht ${ }^{[1]}$. Das vielfältige Reaktionsverhalten dieser Verbindungsklasse gründet sich auf seine vier reaktiven Zentren - Übergangsmetall, Phosphoratom, $\mathrm{M}-\mathrm{P}-\sigma-$ und $\mathrm{P}-\mathrm{C}-\pi$-Bindung die, wie Einzelbeispiele belegen, die Möglichkeit zur Addition am Phosphor-Atom und der $\mathrm{P}=\mathrm{C}$-Einheit ${ }^{[2]}$ oder zur Ausbildung einer Phosphor-Metall-Doppelbindung eröffnen ${ }^{[3]}$. Durch die Einführung eines Metallfragmentes konnte die Reaktivität am Phosphorzentrum derart gesteigert werden, daß ein Wechsel des für Phosphaalkene typischen $[n+$ 2]-Cycloadditionsverhaltens zu einem carbenartigen ( $[n+$ 1]-Cycloaddition) eintritt ${ }^{[4]}$. Derartige oxidative Additionen sind für Phosphanylmetall-Komplexe hinreichend belegt ${ }^{\left[{ }^{[S]}\right.}$.

In der vorliegenden Arbeit werden weitere Methylenphosphanyl-Komplexe von Chrom, Molybdän, Wolfram, Eisen und Nickel vorgestellt und deren Reaktivitäten kommentiert. Die Synthese der Zielverbindungen ist dabei alternativ über eine heterogene Metallierung von Halogenmethylenphosphanen ${ }^{[6]}$ oder die Insertion eines Metallfragments in eine labile Phosphor-Element-Bindung ${ }^{[7]}$ möglich.

\section{Synthesen}

Die Umsetzung der Halogen-substituierten Phosphaalkene $\mathbf{1}$ a, b mit den Alkali-carbonylmetallaten $\mathbf{2} \mathbf{a}-\mathbf{f}$ führt in Toluol, Pentan oder THF bereits unter milden Reaktions- bedingungen $\left(-78\right.$ bis $\left.0^{\circ} \mathrm{C}\right)$ unter Halogenidsubstitution zur Bildung der Methylenphosphanyl-Komplexe 3a-f bzw. 4a-f (Schema 1). Die Produkte sind in guten Ausbeuten isolierbar und mit Ausnahme von 3a, e sowohl in Lösung als auch in kristalliner Form ausgesprochen stabil.

Schema 1
$\mathrm{x}^{\mathrm{P}=\mathrm{c}} \overbrace{\mathrm{R}}^{\mathrm{SiMe}_{3}}$

1a: $\mathrm{R}=\mathrm{SiMe}_{3}, \mathrm{X}=\mathrm{Cl}$

1b: $\mathrm{R}=\mathrm{Ph}, \mathrm{X}=\mathrm{Br}$
(M) K(Na)

$2 a-f$

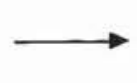

[M]

3a-f: $\mathrm{R}=\mathrm{SiMe}_{3}$

4a-f: $\mathrm{R}=\mathrm{Ph}$
Alternativ dazu lassen sich die Pentamethylcyclopentadienyl-Derivate $\mathbf{3 b}$, d, f ausgehend von dem (Pentamethylcyclopentadienyl)methylenphosphan $\mathbf{5 a}$ über eine formale Insertion eines Metallfragments in die labile $\mathrm{Cp}^{*}-\mathrm{P}$-Bindung aufbauen [Schema 2, Weg (b)]. Ein analoger Prozeß erfolgt bei der Reaktion von $\mathbf{5}$ a mit $(\mathrm{CO})_{3} \mathrm{Cr}\left(\mathrm{CH}_{3} \mathrm{CN}\right)_{3}[\mathrm{Weg}$ (a)] und in Umsetzungen von $\mathbf{5} \mathbf{a}, \mathbf{b}$ mit den in situ erzeugten Nickelkomplexen $7 \mathbf{a}-\mathbf{c}$ [bzw. äquimolaren Mischungen von Bis(cyclooctadien)nickel und einem tertiären Phosphan], die die Chrom- bzw. Nickel-haltigen (Methylenphosphanyl)metall-Komplexe $3 \mathbf{j}$ bzw. $3 \mathrm{~g}-\mathbf{i}, \mathbf{4 g}-\mathbf{i}$ liefern [Weg (c)]. Thermische Reaktion von 5a mit $(\mathrm{CO})_{3} \mathrm{M}\left(\mathrm{CH}_{3} \mathrm{CN}\right)_{3}$ $(\mathrm{M}=\mathrm{Mo}, \mathrm{W})$ führt über die spektroskopisch nachweisba- 
Tab. 1. Metallfragmente $\{\mathbf{M}\}$

\begin{tabular}{|c|c|c|c|}
\hline & (M) & & (M) \\
\hline $2 a, 3 a, 4 a$ & $\mathrm{C}_{5} \mathrm{H}_{5}(\mathrm{CO})_{3} \mathrm{Mo}$ & $2 f, 3 f, 4 f$ & $\mathrm{C}_{5} \mathrm{Me}_{5}(\mathrm{CO})_{2} \mathrm{Fe}$ \\
\hline $2 b, 3 b, 4 b$ & $\mathrm{C}_{5} \mathrm{Me}_{5}(\mathrm{CO})_{3} \mathrm{Mo}$ & $3 \mathrm{~g}, 4 \mathrm{~g}$ & $\mathrm{C}_{5} \mathrm{Me}_{5}\left(\mathrm{Et}_{3} \mathrm{P}\right) \mathrm{Ni}$ \\
\hline $2 c, 3 c, 4 c, 10 a, b, 13-15$ & $\mathrm{C}_{5} \mathrm{H}_{5}(\mathrm{CO})_{3} \mathrm{~W}$ & $3 \mathrm{~h}, 4 \mathrm{~h}$ & $\mathrm{C}_{5} \mathrm{Me}_{5}\left(\mathrm{Bu}_{3} \mathrm{P}\right) \mathrm{Ni}$ \\
\hline $2 d, 3 d, 4 d$ & $\mathrm{C}_{5} \mathrm{Me}_{5}(\mathrm{CO})_{3} \mathrm{~W}$ & $3 i, 4 i, 10 c$ & $\mathrm{C}_{5} \mathrm{Me}_{5}\left(\mathrm{Ph}_{3} \mathrm{P}\right) \mathrm{Ni}$ \\
\hline $2 e, 3 e, 4 e$ & $\mathrm{C}_{5} \mathrm{H}_{5}(\mathrm{CO})_{2} \mathrm{Fe}$ & $3 \mathbf{j}$ & $\mathrm{C}_{5} \mathrm{H}_{5}(\mathrm{CO})_{3} \mathrm{Cr}$ \\
\hline
\end{tabular}

ren Phosphanylmetall-Komplexe $\mathbf{3 b}$, d unter Carbonyl-Eliminierung weiter zu den entsprechenden PhosphavinylidenKomplexen 12b, d d $^{[7]}$ (s. u.).

Die Reaktion wird durch eine Koordination des zunächst noch intakten Phosphaalkens an das Übergangsmetallzentrum eingeleitet ${ }^{[7 \mathrm{~b}]}$. Die resultierenden MethylenphosphanKomplexe sind als Zwischenstufe in den Umsetzungen von 5a, b mit den Nickelkomplexen 7a, b spektroskopisch nachweisbar $(8,9 \mathrm{a}, \mathbf{b})$ oder können bei der Reaktion mit Pentacarbonyleisen unter geeigneten Reaktionsbedingungen isoliert werden $(\mathbf{6 a}, \mathbf{b})$. Die Bildung der (Methylenphosphanyl)metall-Komplexe als Endprodukt wird durch eine anschließende Umlagerung von $\mathbf{6 a}, \mathbf{b}, \mathbf{8}, 9$ unter Verschiebung der Cp*-Gruppe vom Phosphor zum Metall und Abspaltung überzähliger Ko-Liganden vervollständigt. Dieser Reaktionsschritt wird erwartungsgemäß durch das Vorhandensein labiler Austrittsgruppen begünstigt: so erfolgt die Umwandlung der Nickelkomplexe 8, 9a, b in die (Methylenphosphanyl)-Komplexe unter Phosphan-Eliminierung spontan bei Raumtemperatur, während die Decarbonylierung des Eisenkomplexes $\mathbf{6 a}$ erst unter photochemischen Bedingungen zufriedenstellend verläuft ${ }^{[8]}$. In den Reaktionen von $5 \mathrm{a}$, b mit den Acetonitrilkomplexen $(\mathrm{CO})_{3} \mathrm{M}\left(\mathrm{CH}_{3} \mathrm{CN}\right)_{3}$
$(\mathrm{M}=\mathrm{Cr}, \mathrm{Co}, \mathrm{W})$ oder mit $\mathrm{Ni}(\mathrm{COD})_{2} / \mathrm{PR}_{3}$ ist die Umlagerungsreaktion durch die Labilität der Ko-Liganden soweit erleichtert, daß beobachtbare Zwischenstufen fehlen und die Koordination des Methylenphosphans zum geschwindigkeitsbestimmenden Schritt wird. Ein bemerkenswerter Einfluß auf die Stabilität der Zwischenstufen geht zudem von den Substituenten am Methylenkohlenstoff des Liganden aus. Dabei resultiert der formale Ersatz eines Trimethylsilyldurch einen Phenylrest in einer merklichen Stabilisierung der Komplexe, so daß die Umlagerung von $\mathbf{9 a}, \mathbf{b}$ in die entsprechenden (Methylenphosphanyl)nickel-Komplexe signifikant langsamer als im Fall von 8 verläuft und sich der Eisenkomplex $\mathbf{6 b}$ gegenüber einer $\mathrm{CO}$-Eliminierung und Umlagerung zum (Methylenphosphanyl)eisen 4 f sogar als stabil erweist.

Eine der geschilderten Verschiebung eines Cp*-Restes vom Phosphor zum Metall vergleichbare Reaktivität wurde für Halogen-substituierte Methylenphosphane $\mathrm{X}-\mathrm{P}=\mathrm{C}$ $\left(\mathrm{SiMe}_{3}\right)_{2}(\mathrm{X}=\mathrm{Cl}, \mathrm{Br}, \mathrm{I})$ beobachtet, aus denen unter Insertion eines Metallfragments in die labile Phosphor-HalogenBindung ebenfalls Phosphanylmetall-Komplexe zugänglich sind. Dagegen bleibt die Reaktion beim entsprechenden Fluorderivat auf der Stufe der $\eta^{2}$-Komplexe stehen ${ }^{[7 c]}$.

\section{Struktur und Eigenschaften}

Die Konstitution der als Zwischenstufen auftretenden Phosphaalkenkomplexe kann im Fall der äußerst labilen Nickelkomplexe 8, 9a, b aus den ${ }^{31}$ P-NMR-Spektren (ABMSpinsysteme) abgeleitet werden, die für Komplexe mit $\eta^{2}$ koordinierten Methylenphosphanen charakteristische Verschiebungen und Kopplungskonstanten aufweisen ${ }^{[1 b]}$. Für die Eisenkomplexe 6a, b wird sowohl durch IR- als auch NMR-Daten eine isomere Konstitution von $\eta^{1}$-Komplexen

Schema 2

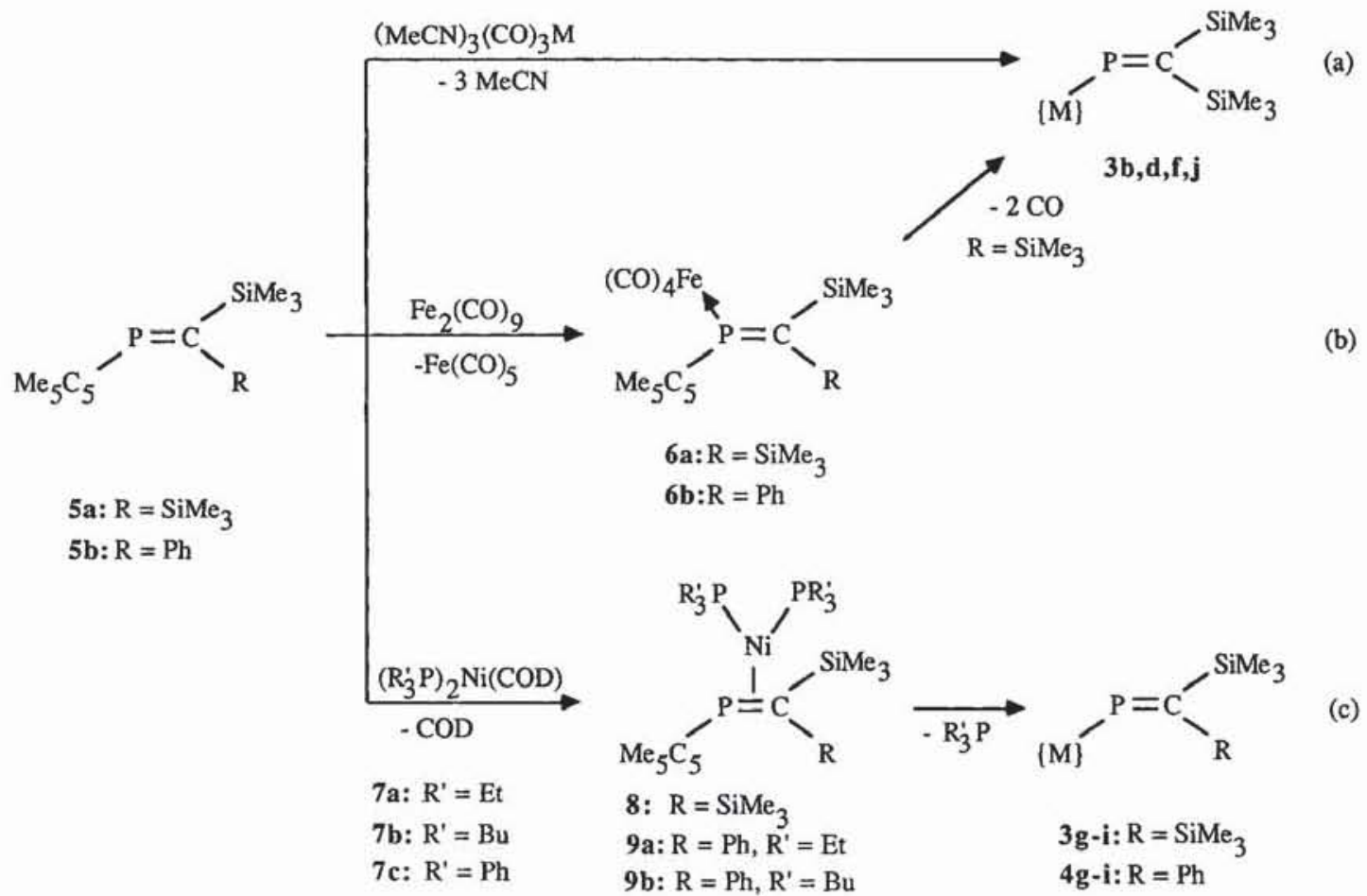


nahegelegt, was für $\mathbf{6 b}$ durch die Ergebnisse einer Röntgenstrukturanalyse bestätigt wird ${ }^{[9]}$.

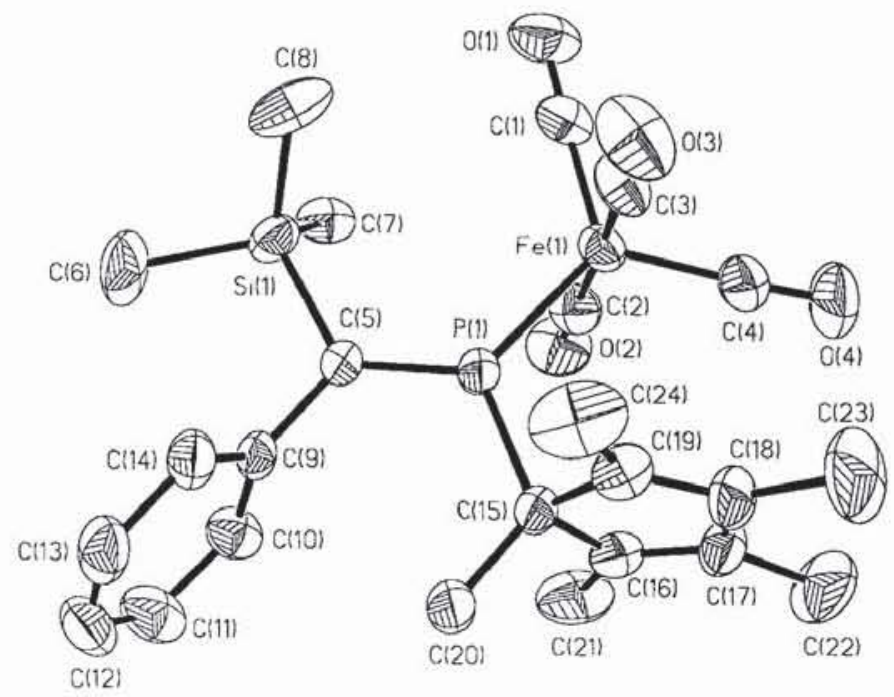

Abb. 1. Kristallstruktur von $\mathbf{6 b}$; Ausgewählte Bindungslängen [pm] und -winkel $\left[{ }^{\circ}\right]$ : $\mathrm{Fe} 1-\mathrm{C} 1$ 176.7(5), $\mathrm{Fe} 1-\mathrm{C} 2$ 178.3(4), $\mathrm{Fe} 1-\mathrm{C} 3$ 179.3(5), Fe1-C4 179.2(5), Fe1-P1 222.5(1), P1-C5 166.4(3), $\mathrm{P} 1-\mathrm{C} 15$ 188.1(4), C15-C16 150.4(5), C16-C17 132.4(6); C1 $\mathrm{Fe} 1-\mathrm{C} 4$ 115.7(2), C1-Fe1-P1 123.8(1), C2-Fe1-P1 86.3(1), $\mathrm{Fe} 1-\mathrm{P} 1-\mathrm{C} 5$ 125.4(1), Fe1-P1-C15 117.8(1), C5-P1-C15 116.5(2), Si1 - C5 - C9 112.1(2)

Die Struktur (Abb. 1) zeigt ein Eisenatom in einer leicht verzerrten, trigonal-bipyramidalen Umgebung, wobei der $\eta^{1}$-Methylenphosphanligand eine für Liganden mit $\pi$-Akzeptoreigenschaften begünstigte äquatoriale Position besetzt und der einer Trimethylsilylgruppe zugewandte $\mathrm{P} 1-\mathrm{Fe}-\mathrm{C} 1-$ Winkel $\left[123.8(1)^{\circ}\right]$ eine Aufweitung auf Kosten des Winkels zwischen den beiden äquatorialen CO-Liganden $\left[\mathrm{C} 1-\mathrm{Fe}-\mathrm{C} 4115.7(2)^{\circ}\right]$ aufweist. Die Phosphor-Kohlenstoff-Doppelbindung im Liganden [166.4(3) pm] ist gegenüber den bekannten Bindungsabständen in $\eta^{1}$-Komplexen leicht erweitert und vergleichbar mit unkomplexierten Methylenphosphanen ${ }^{[1 \mathrm{a}]}$. Im Unterschied zu $\eta^{1}$ $\left[\left(\mathrm{Me}_{3} \mathrm{Si}\right)_{2} \mathrm{C}=\mathrm{PN}\left(\mathrm{SiMe}_{3}\right)_{2}\right] \mathrm{Fe}(\mathrm{CO})_{4}{ }_{4}^{[10]}$ wird für $\mathbf{6 b}$ keine Torsion der Doppelbindung, sondern eine coplanare Anordnung der vier Atome $\mathrm{Si} 1, \mathrm{C} 9, \mathrm{Fe} 1$ und $\mathrm{C} 15$ und damit ein praktisch ungestörtes $\pi$-Bindungssystem beobachtet. Die Cyclopentadienyl-Einheit liegt wie im freien $5 \mathbf{a}^{\mathbf{l 7 a}^{7 a}} \sigma$-gebunden vor, wobei aber die PC-Einfachbindung [188.1(4) pm] als Folge der Komplexierung kontrahiert ist.

Typische Eigenschaften der Metallo-methylenphosphane 3, 4 äußern sich in der starken Entschirmung des ${ }^{31} \mathrm{P}-\mathrm{NMR}$ Resonanzsignals (Tab. 2) sowie im Auftreten extrem langwelliger $n(\mathrm{P})-\pi^{*}(\mathrm{P}=\mathrm{C})$-Übergänge in den UV-VIS-Spektren, die auf eine vergleichsweise niedrige HOMO-LUMODifferenz hindeuten. Beide Effekte sind mit der $\sigma$-Donorwirkung des Metallfragments korrelierbar, die sowohl von der Donorstärke des Übergangsmetalls $\left[\mathrm{W}\left(\mathrm{d}^{4}\right)<\mathrm{Mo}\left(\mathrm{d}^{4}\right)<\right.$ $\left.\mathrm{Fe}\left(\mathrm{d}^{6}\right)<\mathrm{Ni}\left(\mathrm{d}^{8}\right)\right]$ als auch von der der Cyclopentadienyleinheit $\left[\mathrm{Cp}<\mathrm{Cp}^{*}\right]$ abhängig ist. In Verbindungen mit jeweils gleichem Übergangsmetallsubstituenten induziert der formale Ersatz einer Phenyl- durch eine Trimethylsilylgruppe am Methylenkohlenstoff darüber hinaus eine Zunahme von $\delta^{31} \mathrm{P}$ um ca. $100 \mathrm{ppm}$, die dem höheren $\sigma$-Donor/ $\pi$-Akzeptor-Vermögen der Silylgruppe Rechnung trägt ${ }^{[11]}$. Insgesamt können die Substituenteneffekte in den ${ }^{31}$ P-NMR-Spektren, die von Tendenz und Größenordnung den für Organo-substituierte Methylenphosphane beobachteten Effekten vergleichbar sind, wie dort im wesentlichen auf Änderungen des paramagnetischen Verschiebungsbeitrags zurückgeführt werden.

Tab. 2. ${ }^{31} \mathrm{P}-\mathrm{NMR}-\mathrm{Daten}$ von Metallomethylenphosphanen $\{\mathrm{M}\}-\mathrm{P}=\mathrm{C}(\mathrm{R}) \mathrm{SiMe}_{3}$ 3, 4, Metallaphosphaallenen $\{\mathrm{M}\}=\mathrm{P}=\mathrm{C}(\mathrm{R})$ $\mathrm{SiMe}_{3} \mathbf{1 2} \mathbf{a}-\mathbf{d}$ und Komplexen $\{\mathrm{M}\}-\mathrm{P}\left(\mathrm{M}^{\prime} \mathrm{L}_{n}\right)=\mathrm{C}(\mathrm{R}) \mathrm{SiMe}_{3} 11 \mathrm{a}-\mathrm{c}$

\begin{tabular}{|c|c|c|c|c|c|}
\hline Nr. & $\mathbf{R}$ & $\{\mathrm{M}\}$ & $\mathrm{ML}_{\mathrm{n}}$ & $\delta^{31} \mathrm{P}$ & ${ }^{1_{J}}{ }_{\mathrm{PW}}[\mathrm{Hz}]$ \\
\hline $3 \mathrm{a}^{[6]}$ & $\mathrm{SiMe}_{3}$ & $\mathrm{C}_{5} \mathrm{H}_{5}(\mathrm{CO})_{3} \mathrm{Mo}$ & - & 528.5 & - \\
\hline $4 a$ & $\mathrm{Ph}$ & & - & 421.5 & - \\
\hline $3 b^{[6]}$ & $\mathrm{SiMe}_{3}$ & $\mathrm{C}_{5} \mathrm{Me}_{5}(\mathrm{CO})_{3} \mathrm{Mo}$ & - & 588.8 & - \\
\hline $4 b_{161}$ & $\mathrm{Ph}$ & & - & 471.3 & - \\
\hline $3 c^{[6]}$ & $\mathrm{SiMe}_{3}$ & $\mathrm{C}_{5} \mathrm{H}_{5}(\mathrm{CO})_{3} \mathrm{~W}$ & - & 515.2 & 5.0 \\
\hline $4 c$ & $\mathrm{Ph}$ & & - & 403.3 & 4.4 \\
\hline $3 d$ & $\mathrm{SiMe}_{3}$ & $\mathrm{C}_{5} \mathrm{Me}_{5}(\mathrm{CO})_{3} \mathrm{~W}$ & - & 564.3 & 8.8 \\
\hline $4 d$ & $\mathrm{Ph}$ & & - & 441.0 & 9.5 \\
\hline $3 \mathrm{e}^{[2]}$ & $\mathrm{SiMe}_{3}$ & $\mathrm{C}_{5} \mathrm{H}_{5}(\mathrm{CO})_{2} \mathrm{Fe}$ & - & 593.2 & - \\
\hline $4 \mathrm{e}$ & $\mathrm{Ph}$ & & - & 476.3 & - \\
\hline $3 r^{[1]}$ & $\mathrm{SiMe}_{3}$ & $\mathrm{C}_{5} \mathrm{Me}_{5}(\mathrm{CO})_{2} \mathrm{Fe}$ & - & 641.5 & - \\
\hline $4 f$ & $\mathrm{Ph}$ & & - & 520.0 & - \\
\hline $3 g[7 b]$ & $\mathrm{SiMe}_{3}$ & $\mathrm{C}_{5} \mathrm{Me}_{5}\left(\mathrm{Et}_{3} \mathrm{P}\right) \mathrm{Ni}$ & - & $740.5,17.7(\mathrm{~J}=21.3 \mathrm{~Hz})$ & - \\
\hline $4 g$ & $\mathrm{Ph}$ & & - & $619.3,25.7(\mathrm{~J}=14.1 \mathrm{~Hz})$ & - \\
\hline $3 h^{[7 b]}$ & $\mathrm{SiMe}_{3}$ & $\mathrm{C}_{5} \mathrm{Me}_{5}\left(\mathrm{Bu}_{3} \mathrm{P}\right) \mathrm{Ni}$ & - & $740.2,10.3(\mathrm{~J}=23.4 \mathrm{~Hz})$ & - \\
\hline 4h & $\mathrm{Ph}$ & & - & $620.1,16.5(\mathrm{~J}=14.8 \mathrm{~Hz})$ & - \\
\hline $3 \mathrm{i}^{[7 \mathrm{~b}]}$ & $\mathrm{SiMe}_{3}$ & $\mathrm{C}_{5} \mathrm{Me}_{5}\left(\mathrm{Ph}_{3} \mathrm{P}\right) \mathrm{Ni}$ & - & $722.5,39.2(\mathrm{~J}=35.0 \mathrm{~Hz})$ & - \\
\hline $4 i$ & $\mathrm{Ph}$ & & - & $602.3,45.5(\mathrm{~J}=27.9 \mathrm{~Hz})$ & - \\
\hline $3 \mathbf{j}$ & $\mathrm{SiMe}_{3}$ & $\mathrm{C}_{5} \mathrm{H}_{5}(\mathrm{CO})_{3} \mathrm{Cr}$ & - & 605.4 & - \\
\hline 11a & $\mathrm{Ph}$ & $\mathrm{C}_{5} \mathrm{H}_{5}(\mathrm{CO})_{3} \mathrm{~W}$ & $\mathrm{Fe}(\mathrm{CO})_{4}$ & $316.7 / 305.2$ & $78.4 / 86.5$ \\
\hline $11 b$ & $\mathrm{SiMe}_{3}$ & $\mathrm{C}_{5} \mathrm{H}_{5}(\mathrm{CO})_{3} \mathrm{~W}$ & $\mathrm{Ni}(\mathrm{CO})_{3}$ & 423.0 & 417 \\
\hline $11 \mathrm{c}$ & $\mathrm{Ph}$ & $\mathrm{C}_{5} \mathrm{H}_{5}(\mathrm{CO})_{3} \mathrm{~W}$ & $\mathrm{AuCl}$ & 257.9 & 117.2 \\
\hline $12 \mathrm{a}^{[3]}$ & $\mathrm{SiMe}_{3}$ & $\mathrm{C}_{5} \mathrm{H}_{5}(\mathrm{CO})_{2} \mathrm{Mo}$ & - & 497.0 & - \\
\hline $12 \mathrm{~b}$ & $\mathrm{SiMe}_{3}$ & $\mathrm{C}_{5} \mathrm{Me}_{5}(\mathrm{CO})_{2} \mathrm{Mo}$ & - & 493.0 & - \\
\hline $12 \mathrm{c}$ & $\mathrm{SiMe}_{3}$ & $\mathrm{C}_{5} \mathrm{H}_{5}(\mathrm{CO})_{3} \mathrm{~W}$ & - & 446.0 & 617 \\
\hline $12 \mathrm{~d}$ & $\mathrm{SiMe}_{3}$ & $\mathrm{C}_{5} \mathrm{Me}_{5}(\mathrm{CO})_{3} \mathrm{~W}$ & - & 448.0 & 622 \\
\hline
\end{tabular}

Die enge Analogie zwischen Metall- und Organyl-substituierten Methylenphosphanen äußert sich gleichermaßen in den aus Röntgenstrukturanalysen von $\mathbf{3 a}, \mathbf{c}^{[6]}, \mathbf{3} \mathbf{f}^{[8]}, \mathbf{3 b}$, c, $\mathbf{d}^{[6,81}$ erhaltenen Bindungsparametern. Die Doppelbindungslängen [3a 166.5(3) $\left.)^{[6]}, 3 \mathrm{c} 166(2)^{[6]}, 3 \mathrm{f} 168.0(9) \mathrm{pm}^{[8]}\right]$ sowie die übrige Geometrie der $\mathrm{PC}\left(\mathrm{SiMe}_{3}\right)_{2}$-Einheit ist in Übereinstimmung mit der vergleichbarer $P$-Organoderivate ${ }^{[1 a]}$. Demgegenüber erfahren die Valenzwinkel $\mathrm{M}-\mathrm{P}=\mathrm{C}$ eine deutliche Aufweitung [3a 123.5(1) $)^{[6]}$ 3c $125.0(6)^{[6]}$, 3f $\left.126.2(3)^{\circ}{ }^{\circ 8}\right]$, was auf ein Zusammenwirken sowohl sterischer als auch elektronischer Effekte (hohes $\sigma$-Donorvermögen des Metallsubstituenten) zurückgeführt werden kann. Die vergleichsweise kurzen Metall-Phosphorbindungslängen [3f $\left.\mathrm{Fe}-\mathrm{P} 225.6(2) \mathrm{pm}^{[6]}\right]$ gegenüber Methylenphosphankomplexen oder metallsubstituierten Derivaten des Typs $\mathrm{Cp}(\mathrm{CO})_{2} \mathrm{Fe}-\mathrm{P}=\mathrm{C}(\mathrm{R}) \mathrm{OSiMe}_{3}[\mathrm{Fe}-\mathrm{P} 228.9 \mathrm{pm}]^{[12]}$ können wie in Metall-Acyl-Komplexen ${ }^{[13]}$ durch die Annahme einer schwachen Metall-Ligand-Charge-Transfer-Wechselwirkung erklärt werden, die mit dem Auftreten einer entsprechenden Bande im UV-Spektrum vereinbar ist und insgesamt zu einer Erhöhung der Bindungsordnung führen sollte. 
Schema 3

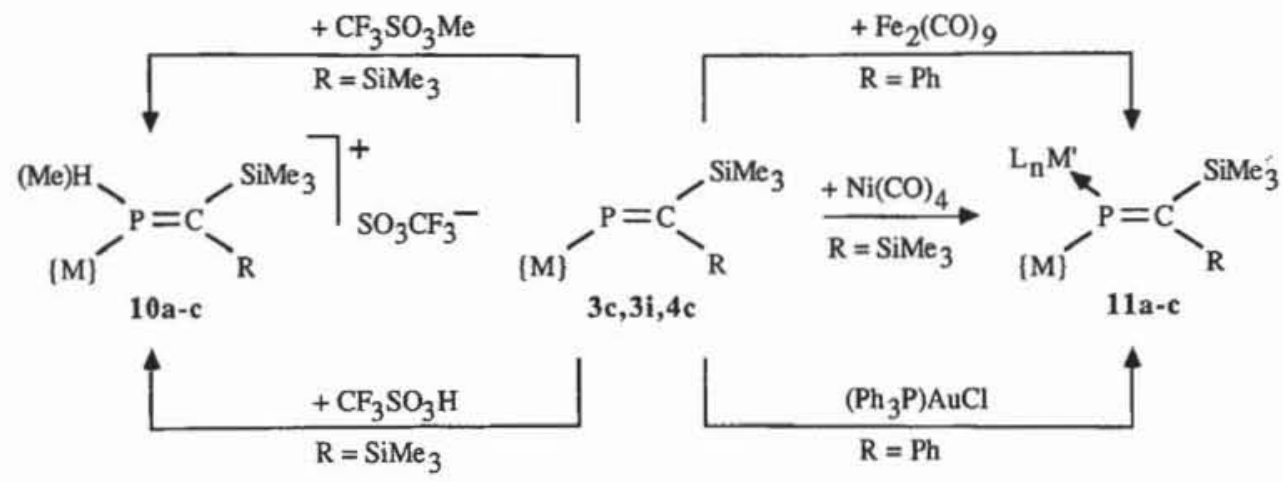

\begin{tabular}{c|ccc} 
& \multicolumn{11}{|c}{$\mathbf{a}$} & \multicolumn{1}{c}{$11 \mathbf{b}$} & \multicolumn{1}{c}{$11 \mathrm{c}$} \\
\hline$\{\mathrm{M}\}$ & $\mathrm{C}_{5} \mathrm{H}_{5}(\mathrm{CO})_{3} \mathrm{~W}$ & $\mathrm{C}_{5} \mathrm{H}_{5}(\mathrm{CO})_{3} \mathrm{~W}$ & $\mathrm{C}_{5} \mathrm{H}_{5}(\mathrm{CO})_{3} \mathrm{~W}$ \\
$\mathrm{~L}_{\mathrm{n}} \mathrm{M}$ & $\mathrm{Fe}(\mathrm{CO})_{4}$ & $\mathrm{Ni}(\mathrm{CO})_{3}$ & $\mathrm{AuCl}$
\end{tabular}

\section{Reaktionen}

Als Folge des hohen Elektronendonorvermögens des Metall-Fragments zeigt der $\lambda^{3}, \sigma^{2}$-Phosphor der (Methylenphosphanyl)metall-Komplexe eine ausgeprägte Nucleophilie, die als wesentliche Ursache für die beobachteten Reaktivitätsmuster (leichte Addierbarkeit von Elektrophilen, Insertions- und Komplexierungsreaktionen) angesehen werden kann.

Anlagerung einer Brønsteadt- oder Lewis-Säure am Phosphorzentrum wird in den Reaktionen von $3 \mathrm{c}$ mit Trifluormethansulfonsäure oder deren Methylester beobachtet, deren Produkte anhand ihrer NMR- und IR-Daten zweifelsfrei als die kationischen Methylenphosphan-Komplexe 10a, b charakterisiert werden können (Schema 3). Die Größe von ${ }^{1} J_{\mathrm{PW}}(\approx 200 \mathrm{~Hz})$ sowic die ${ }^{1} \mathrm{H}-\mathrm{NMR}$-Daten des phosphorständigen Protons in $10 \mathrm{a}\left(\delta=8.91,{ }^{1} J_{\mathrm{PH}}=397 \mathrm{~Hz}\right)$ belegen eine $\eta^{1}$-Koordination der Phosphaalken-Liganden ${ }^{[14]}$. Als ein wesentlicher Beitrag zur Stabilisierung dieser Form kann eine effektive Delokalisation der positiven Ladung über das $\eta^{5}-\mathrm{CpW}(\mathrm{CO})_{3}$-Fragment angesehen werden, die durch die Entschirmung der Cyclopentadienylprotonen $(\Delta \delta=1.4$ gegenüber 3c) sowie eine Erhöhung der $\mathrm{CO}$-Valenzschwingungen angezeigt wird. Dic analoge Reaktion von $\mathbf{3 i} \mathrm{mit}$ einer äquimolaren Menge Trifluormethansulfonsäure bei $-78^{\circ} \mathrm{C}$ liefert das Protonierungsprodukt $10 \mathrm{c}$, das anhand seiner NMR-Daten identifiziert werden kann; eine Isolierung gelang in diesem Fall nicht, bedingt durch die thermische Instabilität und äußerste Oxidationsempfindlichkeit.

In ähnlicher Weise liefern die Reaktionen von $3 \mathbf{c}$ mit $\mathrm{Ni}(\mathrm{CO})_{4}$ und $\mathbf{4 c}$ mit Nonacarbonyldieisen und (Tetrahydrothiophen)gold(I)-chlorid unter Komplexierung der Metallomethylenphosphane die dimetallischen Prodụkte 11a-c, die durch Tieftemperatur-Kristallisation rein isoliert werden können. Während alle Produkte in fester Form bei $20^{\circ} \mathrm{C}$ unter Inertgas lagerfähig sind, unterliegt $\mathbf{1 1}$ b in Lösung innerhalb weniger Stunden einer vollständigen Decarbonylierung unter Bildung des Phosphavinylidenkomplexes 12c (s.u.). Die starke Entschirmung der ${ }^{31} \mathrm{P}$-Signale von 11a-c sowie die gegenüber $\mathbf{3 c}$ signifikant vergrößerten Werte für
${ }^{1} J_{\mathrm{PW}}$ (Tab. 2) sprechen für das Vorliegen von $\mu^{2}(\mathrm{P})-\mathrm{Methy}-$ lenphosphidokomplexen mit einer Koordination beider Metallzentren am Phosphor-Atom. Für 11 a belegen die ${ }^{1} \mathrm{H}$ und ${ }^{29} \mathrm{Si}-\mathrm{NMR}$-Daten die Existenz eines $E / Z$-Isomerenpaars in Lösung.

Im gleichen Zusammenhang kann die aus den (Methylenphosphanyl)metall-Komplexen $\mathbf{3} \mathbf{a}-\mathbf{d}$ beim Erhitzen erfolgende Bildung der Phosphavinyliden-Komplexe 12a-d als Sonderfall einer Komplexierungsreaktion angesehen werden, in der in Analogie zu den Metallo-Phosphanen ${ }^{[5]}$ unter intramolekularer Substitution eines Carbonylliganden eine M/P-Doppelbindung gebildet wird (Schema 4). 12a wurde erstmals von Cowley et al. durch direkte Umsetzung von 1a mit $\mathbf{2} \mathbf{a}$ in THF erhalten ${ }^{[3]}$.

Schema 4

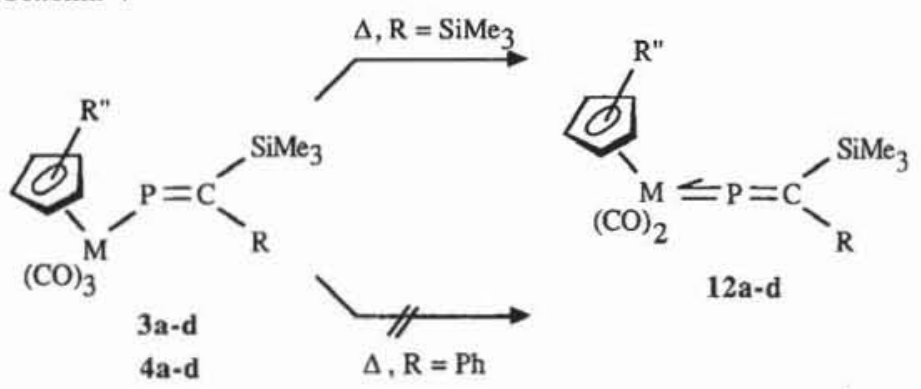

Die zur Abspaltung der CO-Liganden erforderlichen Reaktionsbedingungen orientieren sich an der Stabilität der Metall-CO-Bindung sowie der Elektrophilie des Phosphaalkenylfragments. Dementsprechend setzt die Decarbonylierung der Molybdänderivate $\mathbf{3 a}$, b bereits bei Raumtemperatur in unpolaren Lösungsmitteln ein, während die Bildung der Wolframkomplexe 12c, d mehrstündiges Erhitzen unter Rückfluß in Benzol erfordert. Die Eisenverbindung $3 \mathbf{f}$ sowie die schwächer elektrophilen Phenyl-substituierten (Methylenphosphanyl)metall-Komplexe $\mathbf{4 a - d}$ lassen keine kontrollierte CO-Eliminierung zu; die Thermolyse führt hier ausnahmslos zur Zersetzung der Komplexe. Das Vorliegen eines Heteroallen-Bindungssystems ${ }^{[3]}$ in $12 \mathbf{a}-\mathbf{d}$ äußert sich 
in einer Hochfeldverschiebung der Phosphorsignale und einem starken Anstieg von ${ }^{1} J_{\mathrm{Pw}}$ gegenüber den entsprechenden (Methylenphosphanyl)metall-Komplexen (Tab. 2). Eine gleichzeitige deutliche Abschirmung des Methylenkohlenstoffsignals und eine Abnahme der PC-Kopplungskonstanten implizieren einen erhöhten Elektronenladungstransfer zum Kohlenstoff-Atom hin.

Ein zunächst unerwarteter Verlauf wird für die Reaktionen von 3c mit Schwefel und 3c, 4c mit Selen (jeweils äquimolare Mengen) beobachtet, die nicht zu den erwarteten Oxidationsprodukten 13a-c, sondern unter Insertion des Chalkogens in die Phosphor-Metall-Bindung zu den (Methylenphosphanylthio)- bzw. (Methylenphosphanylseleno)metall-Komplexen 14a $-\mathbf{c}$ führen. Demgegenüber liefert die exzessive Schwefelung von 4c unter Erhalt der PhosphorWolfram-Bindung den $\left(1,2 \lambda^{5}\right.$-Thiaphosphiran-2-yl)wolframKomplex 15.

Schema 5<smiles>[R]C(=PC)[C@H](C)CC</smiles>

13a-d

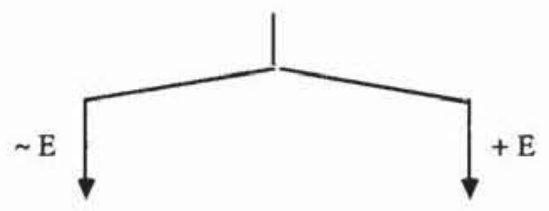<smiles>[R]C(=PCC)SC</smiles>

14a: $\mathrm{R}=\mathrm{SiMe}_{3}, \mathrm{E}=\mathrm{S}$

$14 \mathrm{~b}: \mathrm{R}=\mathrm{SiMe}_{3}, \mathrm{E}=\mathrm{Se}$

$14 \mathrm{c}: \mathrm{R}=\mathrm{Ph}, \mathrm{E}=\mathrm{Se}$<smiles>[R]C1([AsH3])PP1(C)=P</smiles>

$15: R=P h, E=S$

Eine Erklärung dieses Reaktionsverhaltens geht von der Bildung der postulierten Metallochalkogeno(methylen)phosphorane 13a-d als instabile Zwischenstufe aus, die sich unter 1,2-Verschiebung des Metallfragmentes und Bildung einer stabilen Metall-Chalkogen-Bindung in 14a-c umlagern bzw. durch überschüssigen Schwefel unter Bildung von 15 abgefangen werden. Ein vergleichbarer Mechanismus ist für $\sigma^{3}$-Metallophosphan-Systeme bekannt ${ }^{[15]}$. Die Konstitution von $14 \mathbf{a}-\mathbf{c}$ folgt aus der für zweifach koordinierten Phosphor typischen ${ }^{31} \mathrm{P}$-Verschiebung $(\delta=340-417)$, dem geringen Betrag der $J_{\mathrm{PW}}$-Kopplung $(11-14 \mathrm{~Hz})$ sowie den für Einfachbindungen typischen Kopplungen $J_{\mathrm{PSe}}$ (14b 337, $14 \mathrm{c} 326 \mathrm{~Hz})^{[16]} .15$ zeigt neben einer stark abgeschirmten Phosphorresonanz $\left(\delta^{31} \mathrm{P}=-17.7\right)$ eine signifikant höhere $J_{\mathrm{PW}}$-Kopplung $(219.8 \mathrm{~Hz})$, die in der für direkte ${ }^{31} \mathrm{P}^{1}{ }^{183} \mathrm{~W}$ Kopplungen mit tetravalentem Phosphor typischen GröBenordnung liegt ${ }^{[17]}$.

Die dargestellten Methylenphosphan-ÜbergangsmetallKomplexe besitzen strukturell ähnliche Eigenschaften wie analoge Derivate mit organischen Substituenten, zeichnen sich demgegenüber jedoch durch eine gesteigerte Nucleophilie am Phosphor-Atom aus. Die dadurch bedingte „carbenanalogen "[18] Eigenschaften ermöglichen im wesentlichen die Erklärung des beobachteten Reaktivitätsmusters (Addition von Lewis-Säuren, Oxidative Addition, Komplexierung). Die Anwesenheit einer labilen Metall-Phosphor-Bindung erlaubt Chalcogen-Insertionen als interessante Folgereaktion.

Wir danken der Deutschen Forschungsgemeinschaft und dem Fonds der Chemischen Industrie für die Förderung dieser Arbeit. Ein Teil der Arbeiten wurden im Rahmen des SFB 347 (Selektive Reaktionen Metall-aktivierter Moleküle) durchgeführt. H. J. M. dankt dem Deutschen Akademischen Austauschdienst für ein Auslandsstipendium.

\section{Experimenteller Teil}

Alle Arbeiten wurden unter Ausschluß von Luft und Feuchtigkeit unter Inertgas durchgeführt. Die verwendeten Geräte, Chemikalien und Lösungsmittel waren entsprechend vorbereitet. - NMR: ${ }^{31} \mathrm{P}\left\{{ }^{1} \mathrm{H}\right\}$ : Varian FT80A (32.2 MHz) oder Jeol FX 90Q, externer Standard 85proz. $\mathrm{H}_{3} \mathrm{PO}_{4} ;{ }^{13} \mathrm{C}\left\{{ }^{1} \mathrm{H}\right\}$ : Varian FT80A (20 MHz) oder Bruker AM400 (100 MHz), externer Standard TMS; ${ }^{29} \mathrm{Si}\left\{{ }^{1} \mathrm{H}\right\}$ : Varian FT80A (15.6 MHz), externer Standard TMS; ${ }^{1} \mathrm{H}$ : Varian FT80A $(80 \mathrm{MHz})$, Bruker WH $90(90 \mathrm{MHz})$ oder Bruker AM200 $(200 \mathrm{MHz})$, externer Standard TMS. Positives Vorzeichen bedeutet Tieffeldverschiebung relativ zum Standard. Die Aufspaltung von Signalen bezeichnen, wenn nicht anders spezifiziert, Kopplungen mit ${ }^{31}$ P. - MS: VG Instruments VG 12-250 bzw. Kratos MS 50, DirekteinlaB. - UV/VIS: Beckmann Acta M IV (Quarzküvetten, $\mathrm{d}=0.1 \mathrm{~cm}, n$-Heptan, Angabe von $\lambda_{\max }[\mathrm{nm}], \varepsilon_{\max }\left[1 \mathrm{~cm}^{-1} \mathrm{~mol}^{-1}\right]$; $s=$ Schulter). - MS: Die angegebenen Massenzahlen beziehen sich auf das häufigste Isotop des Elements. - Analysen: Gerät Heraeus CHN-O-Rapid.

$\left\{[\right.$ Bis (trimethylsilyl)methylen]phosphanyl $\}$ tricarbonyl $\left(\eta^{5}-c y-\right.$ clopentadienyl)molybdän (3a), \{[Bis(trimethylsilyl) methylen]phosphanyl $\}$ tricarbonyl $\left(\eta^{3}\right.$-pentamethylcyclopentadienyl) molybdän (3b) und $\left\{[\right.$ Bis (trimethylsilyl)methylen]phosphanyl $\}$ tricarbonyl $\left(\eta^{5}-c y-\right.$ clopentadienyl)wolfram (3c): $10 \mathrm{mmol}$ der festen Alkali-tricarbonyl(cyclopentadienyl)metallate $\mathbf{2 a}-\mathbf{c}$ werden unter Rühren in einer Lösung von $2.25 \mathrm{~g} \mathrm{(10} \mathrm{mmol)} 1 \mathrm{a}$ in $40 \mathrm{ml}$ Toluol suspendiert. Die Mischung wird auf $0^{\circ} \mathrm{C}$ gekühlt und mit $2 \mathrm{ml}$ wasserfreiem THF versetzt. Nach $2 \mathrm{~h}$ bei $0^{\circ} \mathrm{C}$ wird auf Raumtemp. erwärmt und weitere $12 \mathrm{~h}$ gerührt. Anschließend werden alle flüchtigen Bestandteile im Hochvak. entfernt, der Rückstand wird zweimal mit $35 \mathrm{ml} \mathrm{He}$ xan extrahiert, und die vereinigten Extrakte werden auf $15-20 \mathrm{ml}$ eingeengt. Kristallisation bei $-80^{\circ} \mathrm{C}$ liefert die Metallomethylenphosphane 3a-c, die abfiltriert und im Hochvak. getrocknet werden.

3b: Methode B: Eine Lösung von $0.65 \mathrm{~g}(2 \mathrm{mmol}) 5 \mathrm{a}$ und $2 \mathrm{mmol}$ 7a in $100 \mathrm{ml}$ Pentan wird in einem Kolben aus Pyrex-Glas $2 \mathrm{~h}$ von außen mit einer Hanau TQ-718 HG-Dampflampe bestrahlt. Unter Gasentwicklung tritt dabei eine Farbvertiefung ein. Die Reaktionslösung wird auf $20 \mathrm{ml}$ eingeengt und filtriert. Kristallisation aus Pentan bei $-80^{\circ} \mathrm{C}$ liefert $0.40 \mathrm{~g} 3 \mathrm{~b}(40 \%)$.

3a: Schmp. $72-74^{\circ} \mathrm{C}$ (Zers.), Ausb. 2.43 g (56\%). - ${ }^{31}$ P-NMR $\left(\mathrm{C}_{6} \mathrm{D}_{6}\right): \delta=528.5$. $-{ }^{13} \mathrm{C}-\mathrm{NMR}\left(\mathrm{C}_{6} \mathrm{D}_{6}\right): \delta=235.3$ (s, trans-CO), 225.4 (d, $J=13.9 \mathrm{~Hz}$, cis-CO), 217.6 (d, $J=109.5 \mathrm{~Hz}, \mathrm{P}=\mathrm{C}$ ), 96.4 $\left(\mathrm{d}, J=4.6 \mathrm{~Hz}, \mathrm{C}_{5} \mathrm{H}_{5}\right), 3.5(\mathrm{~d}, J=15.2 \mathrm{~Hz})$ und $2.9(\mathrm{~s})\left[\mathrm{Si}\left(\mathrm{CH}_{3}\right)_{3}\right]$. $-{ }^{1} \mathrm{H}-\mathrm{NMR}\left(\mathrm{C}_{6} \mathrm{D}_{6}\right): \delta=4.81\left(\mathrm{~d}, J=1.8 \mathrm{~Hz}, 5 \mathrm{H}, \mathrm{C}_{5} \mathrm{H}_{5}\right), 0.55(\mathrm{~s}$, $9 \mathrm{H})$ und $0.35\left(\mathrm{~d}, J=2.4 \mathrm{~Hz}, 9 \mathrm{H}, \mathrm{SiCH}_{3}\right)$. $-\mathrm{MS}, m / z(\%): 436$ 
(1) $\left[\mathrm{M}^{+}\right], 408(16)\left[\mathrm{M}^{+}-\mathrm{CO}\right], 380(10)\left[\mathrm{M}^{+}-2 \mathrm{CO}\right], 352(35)$ $\left[\mathrm{M}^{+}-3 \mathrm{CO}\right], 350(47), 276(22), 73(100)\left[\mathrm{Me}_{3} \mathrm{Si}^{+}\right]$und weitere Bruchstücke. - IR ( $v_{\mathrm{CO}}$, Hexan): $1996 \mathrm{~cm}^{-1}$ st, $1930 \mathrm{~m}, 1915 \mathrm{st} .-$ UV: $\lambda_{\max }\left(\varepsilon_{\max }\right)=450(150, \mathrm{~s}) \mathrm{n}-\pi^{*}, 375(2100, \mathrm{~s}), 316(16400), 251$ (10000).

$$
\mathrm{C}_{15} \mathrm{H}_{23} \mathrm{MoO}_{3} \mathrm{PSi}_{2} \text { (436.0) Ber. C } 41.47 \text { H } 5.33
$$

Gef. C 42.25 H 5.75

3b: Schmp. $74-76^{\circ} \mathrm{C}$ (Zers.), Ausb. $3.83 \mathrm{~g}(76 \%)$ - ${ }^{31} \mathrm{P}-\mathrm{NMR}$ $\left(\mathrm{C}_{6} \mathrm{D}_{6}\right): \delta=588.8 .-{ }^{13} \mathrm{C}-\mathrm{NMR}\left(\mathrm{C}_{6} \mathrm{D}_{6}\right): \delta=237.1(\mathrm{~d}, J=7.9 \mathrm{~Hz}$, trans-CO), 226.3 (d, $J=14.1 \mathrm{~Hz}$, cis-CO), 218.0 (d, $J=106.9 \mathrm{~Hz}$, $\mathrm{P}=\mathrm{C}), 106.5\left[\mathrm{~s}, C_{5}\left(\mathrm{CH}_{3}\right)_{5}\right], 9.9\left[\mathrm{~d}, J=8.7 \mathrm{~Hz}, \mathrm{C}_{5}\left(\mathrm{CH}_{3}\right)_{5}\right], 3.4(\mathrm{~d}, J$ $=15.1 \mathrm{~Hz})$ und $2.8\left[\mathrm{~d}, J=2.5 \mathrm{~Hz}, \mathrm{Si}\left(\mathrm{CH}_{3}\right)_{3}\right] .-\mathrm{MS}, m / z(\%): 478$ (2) $\left[\mathrm{M}^{+}-\mathrm{CO}\right], 422(9)\left[\mathrm{M}^{+}-3 \mathrm{CO}\right], 73(100)\left[\mathrm{Me}_{3} \mathrm{Si}^{+}\right]$und weitere Bruchstücke. - IR ( $v_{\text {co }}$, Hexan): $1992 \mathrm{~cm}^{-1}$ st, $1924 \mathrm{~m}$, 1914 st. - UV: $\lambda_{\max .}\left(\varepsilon_{\max .}\right)=482(240, \mathrm{~s}) \mathrm{n}-\pi^{*}, 385(2200, \mathrm{~s}), 315$ (18500), $252(12400, \mathrm{~s})$.

\section{$\mathrm{C}_{20} \mathrm{H}_{33} \mathrm{MoO}_{3} \mathrm{PSi}_{2}$ (506.1) Ber. C 47.43 H 6.59} Gef. C 46.95 H 6.53

3c: Schmp. $99-101^{\circ} \mathrm{C}$ (Zers.), Ausb. $3.29 \mathrm{~g}(63 \%)$. - ${ }^{31} \mathrm{P}-\mathrm{NMR}$ $\left(\mathrm{C}_{6} \mathrm{D}_{6}\right): \delta=505.2\left(J_{\mathrm{WP}}=5 \mathrm{~Hz},{ }^{1} J_{\mathrm{PC}}=106.2,{ }^{2} J_{\mathrm{PCSi}}=40.0 \mathrm{~Hz}\right)$. $-{ }^{13} \mathrm{C}-\mathrm{NMR}\left(\mathrm{C}_{6} \mathrm{D}_{6}\right): \delta=224.5\left(\mathrm{~d},{ }^{2} J_{\mathrm{CWP}}=7.1 \mathrm{~Hz}\right.$, trans-CO), 215.9 (d, ${ }^{2} J_{\mathrm{CWP}}=9.3 \mathrm{~Hz}$, cis-CO), $210.1\left(\mathrm{~d},{ }^{1} J_{\mathrm{CP}}=106.4 \mathrm{~Hz}, \mathrm{P}=\mathrm{C}\right.$ ), 95.0 $\left(\mathrm{d},{ }^{2} J_{\mathrm{CWP}}=4.5 \mathrm{~Hz}, \mathrm{C}_{5} \mathrm{H}_{5}\right), 3.4\left(\mathrm{~d},{ }^{3} J_{\mathrm{CSiCP}}=15.8 \mathrm{~Hz}\right)$ und $3.0(\mathrm{~d}$, $\left.{ }^{3} J_{\mathrm{CSiCP}}=2.5 \mathrm{~Hz}\right)\left[\mathrm{Si}\left(\mathrm{CH}_{3}\right)_{3}\right] .-{ }^{1} \mathrm{H}-\mathrm{NMR}\left(\mathrm{C}_{6} \mathrm{D}_{6}\right): \delta=4.80\left(\mathrm{~d},{ }^{3} J_{\mathrm{HCWP}}\right.$ $\left.=1.5 \mathrm{~Hz}, 5 \mathrm{H}, \mathrm{C}_{5} \mathrm{H}_{5}\right), 0.56\left[\mathrm{~s}, 9 \mathrm{H}, \mathrm{Si}\left(\mathrm{CH}_{3}\right)_{3}\right], 0.35\left[\mathrm{~d},{ }^{4} J_{\mathrm{HCSiCP}}=2.3\right.$ $\left.\mathrm{Hz}, 9 \mathrm{H}, \mathrm{Si}\left(\mathrm{CH}_{3}\right)_{3}\right] .-{ }^{29} \mathrm{Si}-\mathrm{NMR}\left(\mathrm{C}_{3} \mathrm{D}_{6} \mathrm{O}\right): \delta=30.0\left(\mathrm{~d},{ }^{2} J_{\mathrm{SiCP}}=11.7\right.$ $\mathrm{Hz}, 1 \mathrm{Si}), 29.3\left(\mathrm{~d},{ }^{2} J_{\mathrm{SiCP}}=41.0 \mathrm{~Hz}, 1 \mathrm{Si}\right) .-\mathrm{MS}, m / z(\%): 522(1)$ $\left[\mathrm{M}^{+}\right], 494(10)\left[\mathrm{M}^{+}-\mathrm{CO}, 466(9)\left[\mathrm{M}^{+}-\mathrm{Me},-2 \mathrm{CO}\right], 436(9)\right.$ $\left[\mathrm{M}^{+}-3 \mathrm{CO},-\mathrm{H}_{2}\right], 423$ (9) $\left[\mathrm{M}^{+}-\mathrm{Me},-2 \mathrm{CO}\right], 73$ (100) $\left[\mathrm{Me}_{3} \mathrm{Si}^{+}\right]$. - IR ( $\mathrm{v}_{\mathrm{CO}}$, Hexan): $1992 \mathrm{~cm}^{-1}$ st, $1922 \mathrm{~m}, 1910$ st. UV: $\lambda_{\max .}\left(\varepsilon_{\max }\right)=455(270, \mathrm{~s}) \mathrm{n}-\pi^{*}, 370(2300, \mathrm{~s}), 310(11900), 245$ $(8600, \mathrm{~s})$.

$$
\mathrm{C}_{15} \mathrm{H}_{23} \mathrm{O}_{3} \mathrm{PSi}_{2} \mathrm{~W} \text { (522.0) Ber. C } 34.49 \mathrm{H} 4.44
$$

\section{Gef. C 34.37 H 4.36}

$\left\{\left[\right.\right.$ Bis (trimethylsilyl) methylen] phosphanyl\}tricarbonyl ( $\eta^{5}$-pentamethylcyclopentadienyl) wolfram (3d): Eine Suspension von $2.74 \mathrm{~g}$ $(7.7 \mathrm{mmol}) 2 \mathrm{~d}$ in $60 \mathrm{ml}$ Petrolether $\left(30-70^{\circ} \mathrm{C}\right)$ wird bei $-78^{\circ} \mathrm{C}$ mit $1.73 \mathrm{~g}$ (7.7 mmol) 1 a versetzt. Nach Erwärmen auf Raumtemp. wird $2 \mathrm{~h}$ gerührt, dann wird Unlösliches abgetrennt, das Solvens i. Vak. bis auf $5 \mathrm{ml}$ eingeengt, $3 \mathrm{~d}$ bei $-78^{\circ} \mathrm{C}$ ausgefroren, abfiltriert und i. Vak. getrocknet. Ausb. $2.80 \mathrm{~g}(70 \%)$, orangefarbenes Kristallpulver, Schmp. $110^{\circ} \mathrm{C}$ (Zers.).

Methode B: Eine Lösung von $1.23 \mathrm{~g}(2.5 \mathrm{mmol}) \mathbf{5} \mathrm{a}$ und $2.5 \mathrm{mmol}$ $(\mathrm{MeCN})_{3}(\mathrm{CO})_{3} \mathrm{~W}$ in $150 \mathrm{ml}$ Hexan wird $2-3 \mathrm{~h}$ mit einer $\mathrm{Hg}-$ Dampflampe bestrahlt. Die Lösung wird i. Vak. auf $30 \mathrm{ml}$ eingeengt, das Produkt abfiltriert und im Hochvak. getrocknet. Ausb. $0.78 \mathrm{~g}$ $(53 \%) 3 d$

${ }^{31} \mathrm{P}-\mathrm{NMR}\left(\mathrm{C}_{3} \mathrm{D}_{6} \mathrm{O}\right): \delta=564.3\left({ }^{1} J_{\mathrm{WP}}=8.8,{ }^{2} J_{\mathrm{PCSi}}=38.1 \mathrm{~Hz}\right)$. ${ }^{13} \mathrm{C}-\mathrm{NMR}\left(\mathrm{C}_{6} \mathrm{D}_{6}\right): \delta=227.4\left(\mathrm{~d}, J_{\mathrm{CWP}}=8.9 \mathrm{~Hz}\right.$, trans-CO), 218.2 $\left(\mathrm{d},{ }^{2} J_{\mathrm{CWP}}=10.1,{ }^{1} J_{\mathrm{WC}}=156.8 \mathrm{~Hz}\right.$, cis-CO), $210.6\left(\mathrm{~d},{ }^{1} J_{\mathrm{CP}}=105.0\right.$ $\mathrm{Hz}, \mathrm{P}=\mathrm{C}), 105.5\left[\mathrm{~s}, \mathrm{C}_{5}\left(\mathrm{CH}_{3}\right)_{5}\right], 10.1\left[\mathrm{~d},{ }^{3} J_{\mathrm{CCWP}}=8.7 \mathrm{~Hz}, \mathrm{C}_{5}\left(\mathrm{CH}_{3}\right)_{5}\right]$, $3.7\left[\mathrm{~d},{ }^{3} J_{\mathrm{CSiCP}}=15.5 \mathrm{~Hz}, \mathrm{Si}\left(\mathrm{CH}_{3}\right)_{3}\right], 3.0\left[\mathrm{~d},{ }^{3} J_{\mathrm{CSiCP}}=38.1 \mathrm{~Hz}\right.$, $\left.\mathrm{Si}\left(\mathrm{CH}_{3}\right)_{3}\right] .{ }^{1} \mathrm{H}-\mathrm{NMR}\left(\mathrm{C}_{6} \mathrm{D}_{6}\right): \delta=1.72\left[\mathrm{~s}, 15 \mathrm{H}, \mathrm{C}_{5}\left(\mathrm{CH}_{3}\right)_{5}\right], 0.53[\mathrm{~s}$, $\left.9 \mathrm{H}, \mathrm{Si}\left(\mathrm{CH}_{3}\right)_{3}\right], 0.36\left[\mathrm{~d},{ }^{4} J_{\mathrm{HCSiCP}}=2.1 \mathrm{~Hz}, 9 \mathrm{H}, \mathrm{Si}\left(\mathrm{CH}_{3}\right)_{3}\right] .-{ }^{29} \mathrm{Si}-$ $\operatorname{NMR}\left(\mathrm{C}_{3} \mathrm{D}_{6} \mathrm{O}\right): \delta=-3.1\left(\mathrm{~d},{ }^{2} J_{\mathrm{SiCP}}=13.2 \mathrm{~Hz}, 1 \mathrm{Si}\right),-3.8\left[\mathrm{~d},{ }^{2} J_{\mathrm{SiC}}\right.$ $\left.=1.9 \mathrm{~Hz}, 1 \mathrm{Si}, \mathrm{Si}\left(\mathrm{CH}_{3}\right)_{3}\right] .-\mathrm{IR}\left(v_{\mathrm{CO}}\right.$, Petrolether $\left.50-70^{\circ} \mathrm{C}\right): 1990$ $\mathrm{cm}^{-1}$ vs, $1918 \mathrm{~s}, 1909$ vs.

$$
\begin{array}{ll}
\mathrm{C}_{20} \mathrm{H}_{33} \mathrm{WO}_{3} \mathrm{PSi}_{2} \text { (592.1) } & \text { Ber. C } 40.53 \text { H } 5.62 \\
& \text { Gef. C } 40.23 \text { H } 5.55
\end{array}
$$

$\left\{\left[\right.\right.$ Bis (trimethylsilyl) methylen]phosphanyl\}dicarbonyl $\left(\eta^{5}-\right.$ cyclopentadienyl)eisen (3e): $\mathrm{Zu}$ einer Lösung von $5 \mathrm{mmol} 2 \mathrm{e}$ in $70 \mathrm{ml}$ THF werden langsam bei $-78^{\circ} \mathrm{C} 1.12 \mathrm{~g}(5 \mathrm{mmol}) 1 \mathrm{a}$ getropft. Es wird innerhalb von $3 \mathrm{~h}$ auf $22^{\circ} \mathrm{C}$ erwärmt und weitere $2 \mathrm{~h}$ bei dieser Temp. gerührt, zur Trockene eingeengt und der Rückstand in $50 \mathrm{ml}$ Pentan aufgenommen. Der Niederschlag wird abgetrennt, und anschließend werden alle flüchtigen Bestandteile im Hochvak. entfernt. Das tiefrote Öl zersetzt sich selbst bei tiefen Temperaturen innerhalb von wenigen Tagen. - ${ }^{31} \mathrm{P}-\mathrm{NMR}\left(\mathrm{C}_{6} \mathrm{D}_{6}\right): \delta=593.2$. ${ }^{13} \mathrm{C}-\mathrm{NMR}\left(\mathrm{C}_{6} \mathrm{D}_{6}\right): \delta=215.2\left(\mathrm{~d}, J_{\mathrm{CFeP}}=3.6 \mathrm{~Hz}, \mathrm{CO}\right), 211.2(\mathrm{~d}, J=$ $110.9 \mathrm{~Hz}, \mathrm{P}=\mathrm{C}), 88.5\left(\mathrm{~d}, J=4.3 \mathrm{~Hz}, \mathrm{C}_{5} \mathrm{H}_{5}\right), 3.9[\mathrm{~d}, J=12.4 \mathrm{~Hz}$, $\left.\mathrm{Si}\left(\mathrm{CH}_{3}\right)_{3}\right], 3.5\left[\mathrm{~d}, J=6.5 \mathrm{~Hz}, \mathrm{Si}\left(\mathrm{CH}_{3}\right)_{3}\right] .-{ }^{1} \mathrm{H}-\mathrm{NMR}\left(\mathrm{C}_{6} \mathrm{D}_{6}\right): \delta=$ $4.19\left(\mathrm{~d}, J=2.3 \mathrm{~Hz}, 5 \mathrm{H}, \mathrm{C}_{5} \mathrm{H}_{5}\right), 0.57\left[\mathrm{~s}, 9 \mathrm{H}, \mathrm{Si}\left(\mathrm{CH}_{3}\right)_{3}\right], 0.42[\mathrm{~d}, J=$ $\left.2.6 \mathrm{~Hz}, 9 \mathrm{H}, \mathrm{Si}\left(\mathrm{CH}_{3}\right)_{3}\right] .-\mathrm{MS}, m / z(\%): 366$ (2) $\left[\mathrm{M}^{+}\right], 338$ (4) $\left[\mathrm{M}^{+}-\mathrm{CO}\right.$ ], $310(15)\left[\mathrm{M}^{+}-2 \mathrm{CO}, 73(100)\left[\mathrm{SiMe}_{3}^{+}\right]\right.$und weitere Bruchstücke.

$\left\{\left[\right.\right.$ Bis (trimethylsilyl ) methylen] phosphanyl\}dicarbonyl $\left(\eta^{5}\right.$-pentamethylcyclopentadienyl)eisen (3f)

Methode A: Eine auf $-70^{\circ} \mathrm{C}$ gekühlte Lösung von $3 \mathrm{mmol} 2 \mathrm{f}$ in einem Lösungsmittelgemisch bestehend aus $25 \mathrm{ml}$ Petrolether (Siedebereich $\left.40-60^{\circ} \mathrm{C}\right)$ und $2 \mathrm{ml}$ THF wird mit $0.67 \mathrm{~g}(3 \mathrm{mmol}) 1$ a versetzt. Nachdem auf Raumtemp. erwärmt wurde, wird weitere $2 \mathrm{~h}$ gerührt, zur Trockene eingeengt und der Rückstand in Pentan gelöst. Kristallisation aus Pentan liefert bei $-80^{\circ} \mathrm{C} 3 \mathrm{f}$ mit $70 \%$ Ausb. (0.92 g).

Methode B: Eine Lösung von $1.23 \mathrm{~g}(2.5 \mathrm{mmol}) 5 \mathrm{a}$ und $2.5 \mathrm{mmol}$ $\mathrm{Fe}_{2}(\mathrm{CO})_{9}$ in $120 \mathrm{ml}$ Hexan wird in einem Kolben aus Pyrex-Glas von außen mit einer Hanau TQ-718 Hg-Dampflampe bestrahlt, wobei unter Gasentwicklung eine langsame Farbänderung nach rotbraun eintritt. Die Reaktion ist nach $3-4$ h beendet (IR-Kontrolle). Die Reaktionslösung wird auf $25 \mathrm{ml}$ eingeengt und filtriert. Das Filtrat wird über eine kurze Säule $(5 \mathrm{~cm}$, mit Triethylamin vorbehandeltes Kieselgel, Hexan) chromatographiert. Nach Einengen der roten Fraktion des Eluats i. Vak. verbleibt ein braunrotes $\mathrm{Ol}$, das spektroskopisch als 3 f identifiziert wird. Ausb. $0.38 \mathrm{~g}(35 \%)$. - ${ }^{31} \mathrm{P}-\mathrm{NMR}\left(\mathrm{C}_{6} \mathrm{D}_{6}\right): \delta=641.5 .-{ }^{13} \mathrm{C}$-NMR $\left(\mathrm{C}_{6} \mathrm{D}_{6}\right): \delta=217.6(\mathrm{~s}$, $\mathrm{CO}), 208.9(\mathrm{~d}, J=106.1 \mathrm{~Hz}, \mathrm{P}=\mathrm{C}), 98.9\left[\mathrm{~s}, C_{5}\left(\mathrm{CH}_{3}\right)_{5}\right], 9.3$ [d, $J=$ $\left.9.2 \mathrm{~Hz}, \mathrm{C}_{5}\left(\mathrm{CH}_{3}\right)_{5}\right], 3.9(\mathrm{~d}, J=15.6 \mathrm{~Hz})$ und $3.5(\mathrm{~s})\left[\mathrm{Si}\left(\mathrm{CH}_{3}\right)_{3}\right] .-\mathrm{MS}$, $m / z(\%): 436(3)\left[\mathrm{M}^{+}\right], 408(44)\left[\mathrm{M}^{+}-\mathrm{CO}\right], 380(100)\left[\mathrm{M}^{+}-2\right.$ CO], $73(73)\left[\mathrm{Me}_{3} \mathrm{Si}^{+}\right]$und weitere Bruchstücke. - IR $\left(v_{\mathrm{CO}}\right.$, Hexan): $1988 \mathrm{~cm}^{-1}$ st, $1942 \mathrm{st}$.

$$
\begin{array}{ll}
\mathrm{C}_{19} \mathrm{H}_{33} \mathrm{FeO}_{2} \mathrm{PSi}_{2} \text { (436.1) } & \text { Ber. C } 52.28 \text { H } 7.63 \\
& \text { Gef. C } 51.99 \text { H } 7.56
\end{array}
$$

$\left\{[\right.$ Bis(trimethylsilyl) methylen]phosphanyl $\}\left(\eta^{5}\right.$-pentamethylcyclopentadienyl)(triethylphosphan)nickel (3g), \{[Bis(trimethylsilyl)methylen]phosphanyl $\}\left(\eta^{5}\right.$-pentamethylcyclopentadienyl)(tributylphosphan) nickel (3h) und \{[Bis(trimethylsilyl) methylen] phosphanyl\} $\left(\eta^{5}\right.$ pentamethylcyclopentadienyl)(triphenylphosphan)nickel (3i). a) Eine Suspension von $0.55 \mathrm{~g} \mathrm{(2} \mathrm{mmol)} \mathrm{Bis(cyclooctadien)nickel} \mathrm{in}$ $2.5 \mathrm{ml}$ Benzol wird unter Rühren mit $2 \mathrm{mmol}$ eines tertiären Phosphans $(0.236 \mathrm{~g}$ Triethylphosphan, $0.404 \mathrm{~g}$ Tri-n-butylphosphan, $0.524 \mathrm{~g}$ Triphenylphosphan) versetzt und anschließend gerührt, bis eine klare, hellgelbe Lösung entstanden ist. - b) Eine Lösung von $0.65 \mathrm{~g} \mathrm{(} 2 \mathrm{mmol}) 5 \mathrm{a}$ in $2 \mathrm{ml}$ Benzol wird unter Rühren mit der nach a) erzeugten Lösung versetzt, wobei eine spontane Reaktion unter Rotfärbung beobachtet wird. Es wird $1 \mathrm{~h}$ nachgerührt; anschließend werden alle i.Vak. bis $35^{\circ} \mathrm{C}$ flüchtigen Bestandteile entfernt. Umkristallisation des Rückstands aus Ether liefert reines $\mathbf{3 i}$ in tiefroten Kristallen; im Fall von $3 \mathrm{~g}$, h gelang eine entsprechende Reinigung nicht, so daß lediglich die als rotbraune Öle anfallenden Rohprodukte charakterisiert werden konnten.

$3 \mathrm{~g}:{ }^{31} \mathrm{P}-\mathrm{NMR}\left(\mathrm{C}_{6} \mathrm{D}_{6}\right): \delta=740.5(\mathrm{~d}, J=21.3 \mathrm{~Hz}, \mathrm{P}=\mathrm{C}), 17.7(\mathrm{~d}$ $\left.J=21.3 \mathrm{~Hz}, \mathrm{PR}_{3}\right) .-{ }^{31} \mathrm{C}-\mathrm{NMR}\left(\mathrm{C}_{6} \mathrm{D}_{6}\right): \delta=207(\mathrm{~d}, J=112.8 \mathrm{~Hz}$, $\mathrm{P}=\mathrm{C}), 99.5\left[\mathrm{~d}, J=1.1 \mathrm{~Hz}, C_{5}\left(\mathrm{CH}_{3}\right)_{5}\right], 16.9\left(\mathrm{dd}, J=25.6 \mathrm{~Hz}, J_{\mathrm{CP}}\right.$ 
$\left.=3.0 \mathrm{~Hz}, \mathrm{PCH}_{2}\right), 10.1\left[\mathrm{~d}, J=5.1 \mathrm{~Hz}, \mathrm{C}_{5}\left(\mathrm{CH}_{3}\right)_{5}\right], 7.5\left(\mathrm{br}, \mathrm{PCCH}_{3}\right)$, $3.7(\mathrm{~d}, J=13.0 \mathrm{~Hz})$ und $2.8(\mathrm{~d}, J=1.6 \mathrm{~Hz})\left[\mathrm{Si}\left(\mathrm{CH}_{3}\right)_{3}\right] .-{ }^{1} \mathrm{H}-\mathrm{NMR}$ $\left(\mathrm{C}_{6} \mathrm{D}_{6}\right): \delta=1.71\left[\mathrm{~s}, 15 \mathrm{H}, \mathrm{C}_{5}\left(\mathrm{CH}_{3}\right)_{5}\right], 1.15\left(\mathrm{~m}, \mathrm{br}, 6 \mathrm{H}, \mathrm{PCH}_{2}\right), 0.81$ $\left(\mathrm{m}, \mathrm{br}, 9 \mathrm{H}, \mathrm{PCCH}_{3}\right), 0.66(\mathrm{~s}, 9 \mathrm{H})$ und $\left.0.45(\mathrm{~s}, 9 \mathrm{H})\left[\mathrm{Si}\left(\mathrm{CH}_{3}\right)_{3}\right)\right]$.

3h: ${ }^{31} \mathrm{P}-\mathrm{NMR}\left(\mathrm{C}_{6} \mathrm{D}_{6}\right): \delta=740.2(\mathrm{~d}, J=23.4 \mathrm{~Hz}, \mathrm{P}=\mathrm{C}), 10.3$ (d, $\left.J=23.4 \mathrm{~Hz}, \mathrm{PR}_{3}\right) .-{ }^{13} \mathrm{C}-\mathrm{NMR}\left(\mathrm{C}_{6} \mathrm{D}_{6}\right): \delta=206.7(\mathrm{~d}, J=111.2$ $\mathrm{Hz}, \mathrm{P}=\mathrm{C}), 99.5\left[\mathrm{~d}, J=0.7 \mathrm{~Hz}, C_{5}\left(\mathrm{CH}_{3}\right)_{5}\right], 26.1\left(\mathrm{~s}, \mathrm{PCCH}_{2}\right), 25.2(\mathrm{~d}$, $\left.J=25.0 \mathrm{~Hz}, \mathrm{PCH}_{2}\right), 24.7\left(\mathrm{~d}, J=12.5 \mathrm{~Hz}, \mathrm{PCCCH}_{2}\right), 14.0(\mathrm{~s}$, $\left.\mathrm{PCCCCH}_{3}\right), 10.2\left[\mathrm{~d}, J=5.8 \mathrm{~Hz}, \mathrm{C}_{5}\left(\mathrm{CH}_{3}\right)_{5}\right], 3.7(\mathrm{~d}, 12.7 \mathrm{~Hz})$ und 2.9 (s) $\left[\mathrm{Si}\left(\mathrm{CH}_{3}\right)_{3}\right] .-{ }^{1} \mathrm{H}-\mathrm{NMR}\left(\mathrm{C}_{6} \mathrm{D}_{6}\right): \delta=1.76\left[\mathrm{~s}, 15 \mathrm{H}, \mathrm{C}_{5}\left(\mathrm{CH}_{3}\right)_{5}\right], 1.40$ (m, br, 6H, PCH $)_{2}, 1.28\left(\mathrm{~m}, \mathrm{br}, 12 \mathrm{H}, \mathrm{PCCH}_{2} \mathrm{CH}_{2}\right), 0.88(\mathrm{~m}, \mathrm{br}, 9 \mathrm{H}$, $\left.\mathrm{PCCCCH}_{3}\right), 0.69(\mathrm{~s}, 9 \mathrm{H})$ und $0.47(\mathrm{~s}, 9 \mathrm{H})\left[\mathrm{Si}\left(\mathrm{CH}_{3}\right)_{3}\right] .-\mathrm{MS}, \mathrm{m} / z$ $(\%): 584(100)\left[\mathrm{M}^{+}\right], 202(5)\left[n \mathrm{Bu}_{3} \mathrm{P}^{+}\right]$und weitere Bruchstücke.

3i: Schmp. $114-119^{\circ} \mathrm{C}$ (Zers.), Ausb. $35-40 \%$. - ${ }^{31} \mathrm{P}-\mathrm{NMR}$ $\left(\mathrm{C}_{6} \mathrm{D}_{6}\right): \delta=722.5(\mathrm{~d}, J=35.0 \mathrm{~Hz}, \mathrm{P}=\mathrm{C}), 39.2(\mathrm{~d}, J=35.0 \mathrm{~Hz}$, $\mathrm{PR}_{3}$ ). $-{ }^{13} \mathrm{C}-\mathrm{NMR}\left(\mathrm{C}_{6} \mathrm{D}_{6}\right): \delta=203.7(\mathrm{dd}, J=109.8, J=3.0 \mathrm{~Hz}$, $\mathrm{P}=\mathrm{C}), 134.5(\mathrm{~d}, J=11.3 \mathrm{~Hz}, \mathrm{C}-2,6), 134.0(\mathrm{~d}, J=41.5 \mathrm{~Hz}, \mathrm{C}-1)$, 129.5 (s, C-4), 128.8 (s, C-3,5), 100.9 [s, $\left.\mathrm{C}_{5}\left(\mathrm{CH}_{3}\right)_{5}\right], 10.0\left[\mathrm{~s}, \mathrm{C}_{5}\left(\mathrm{CH}_{3}\right)_{5}\right.$, $3.9(\mathrm{~d}, J=11.6 \mathrm{~Hz})$ und $2.9(\mathrm{~s})\left[\mathrm{Si}\left(\mathrm{CH}_{3}\right)_{3}\right] .-{ }^{1} \mathrm{H}-\mathrm{NMR}\left(\mathrm{C}_{6} \mathrm{D}_{6}\right): \delta=$ $7.52(\mathrm{~m}, 6 \mathrm{H})$ und $7.02(\mathrm{~m}, 9 \mathrm{H})\left(\mathrm{PC}_{6} \mathrm{H}_{5}\right), 1.56[\mathrm{~d}, J=0.8 \mathrm{~Hz}, 15 \mathrm{H}$, $\left.\mathrm{C}_{5}\left(\mathrm{CH}_{3}\right)_{5}\right], 0.54(\mathrm{~s}, 9 \mathrm{H})$ und $0.32(\mathrm{~s}, 9 \mathrm{H})\left[\mathrm{Si}\left(\mathrm{CH}_{3}\right)_{3}\right] .-\mathrm{UV}: \lambda_{\max ,}\left(\varepsilon_{\max }\right)$ $=640(330, \mathrm{~s}) \mathrm{n}-\pi^{*}, 454(4600), 375(4700), 331(6900, \mathrm{~s}), 302(\mathrm{~s})$, 274 (16300), 257 (s).

$$
\begin{array}{ll}
\mathrm{C}_{35} \mathrm{H}_{48} \mathrm{NiP}_{2} \mathrm{Si}_{2} \text { (644.2) } & \text { Ber. C } 65.12 \text { H } 7.49 \\
& \text { Gef. C } 65.00 \text { H } 7.50
\end{array}
$$

$\left\{[\right.$ Bis(trimethylsilyl) methylen]phosphanyl $\}$ tricarbonyl $\left(\eta^{5}\right.$-pentamethylcyclopentadienyl)chrom (3j): Eine Mischung von $2.64 \mathrm{~g} \mathrm{(5}$ $\mathrm{mmol}) 5 \mathrm{a}$ und $1.35 \mathrm{~g}(5 \mathrm{mmol})(\mathrm{MeCN})_{3} \mathrm{Cr}(\mathrm{CO})_{3}$ wird in $40 \mathrm{ml}$ Toluol bis zur Vervollständigung der Reaktion ( ${ }^{31} \mathrm{P}-\mathrm{NMR}-\mathrm{Kon}-$ trolle) unter Rückfluß erhitzt. Nach Abkühlen auf Raumtemp. werden flüchtige Bestandteile i. Vak. entfernt, der Rückstand wird zweimal mit $20 \mathrm{ml}$ Hexan extrahiert, und die vereinigten Extrakte werden auf ca. $15 \mathrm{ml}$ eingeengt. Nach Kristallisation bei $-35^{\circ} \mathrm{C}$,

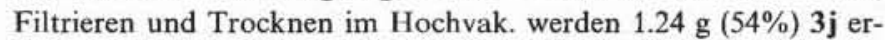
halten. Das Produkt ist selbst bei $-20^{\circ} \mathrm{C}$ unter Inertgas instabil und zerfällt innerhalb kurzer Zeit unter Bildung von $\mathrm{Cr}(\mathrm{CO})_{6}$ sowie unidentifizierter grünlicher Zersetzungsprodukte. Schmp. $63-74^{\circ} \mathrm{C}$ (Zers.). - ${ }^{31} \mathrm{P}-\mathrm{NMR}\left(\mathrm{C}_{6} \mathrm{D}_{6}\right): \delta=605.4 .-{ }^{13} \mathrm{C}-\mathrm{NMR}\left(\mathrm{C}_{6} \mathrm{D}_{6}\right): \delta=$ 245.2 (d, $J=1.3 \mathrm{~Hz}$, trans-CO), 235.5 (d, $J=21.4 \mathrm{~Hz}$, cis-CO), $222.2(\mathrm{~d}, J=107.6 \mathrm{~Hz}, \mathrm{P}=\mathrm{C}), 102.2\left[\mathrm{~s}, C_{5}\left(\mathrm{CH}_{3}\right)_{5}\right], 9.3[\mathrm{~d}, J=9.7$ $\left.\mathrm{Hz}, \mathrm{C}_{5}\left(\mathrm{CH}_{3}\right)_{5}\right], 3.4(\mathrm{~d}, J=15.5 \mathrm{~Hz})$ und $2.5(\mathrm{~d}, J=2.3 \mathrm{~Hz})\left[\mathrm{Si}\left(\mathrm{CH}_{3}\right)_{3}\right]$. - ${ }^{\prime} \mathrm{H}$-NMR $\left(\mathrm{C}_{6} \mathrm{D}_{6}\right): \delta=1.53\left[\mathrm{~s}, 15 \mathrm{H}, \mathrm{C}_{5}\left(\mathrm{CH}_{3}\right)_{5}\right], 0.56(\mathrm{~s}, 9 \mathrm{H})$ und $0.38(\mathrm{~d}, J=2.4 \mathrm{~Hz}, 9 \mathrm{H})\left[\mathrm{Si}\left(\mathrm{CH}_{3}\right)_{3}\right] .-\mathrm{MS}, m / z(\%): 432(11)\left[\mathrm{M}^{+}\right.$ - CO], $376(42)\left[\mathrm{M}^{+}-3 \mathrm{CO}, 187(74), 73(100)\left[\mathrm{Me}_{3} \mathrm{Si}^{+}\right]\right.$und weitere Bruchstücke. - IR ( $v_{\mathrm{CO}}$, Hexan): $1975 \mathrm{~cm}^{-1}$ sst, 1916 st, 1900 sst.

Tricarbonyl ( $\eta^{5}$-cyclopentadienyl) \{[methyl(triphenylsilyl) methylen]phosphanyl'molybdän (4a): Eine Lösung von $3.5 \mathrm{mmol} \mathbf{2 a}$ in $35 \mathrm{ml}$ THF wird bei $-50^{\circ} \mathrm{C}$ mit $0.96 \mathrm{~g}(83.5 \mathrm{mmol}) 1 \mathrm{~b}$ versetzt. Es wird $1 \mathrm{~h}$ bei dieser Temp. gerührt, anschließend auf Raumtemp. erwärmt und weitere $2 \mathrm{~h}$ gerührt. Das Lösungsmittel wird i.Vak. entfernt, der gelborangefarbene Rückstand in $30 \mathrm{ml}$ Pentan aufgenommen und die Lösung filtriert. Kristallisation bei $-25^{\circ} \mathrm{C}$ liefert ein orangegelbes Kristallpulver, Schmp. $71^{\circ} \mathrm{C}$, Ausb. $1.30 \mathrm{~g}$ $(85 \%)$ - ${ }^{31} \mathrm{P}-\mathrm{NMR}\left(\mathrm{C}_{6} \mathrm{D}_{6}\right): \delta=421.5 .-{ }^{13} \mathrm{C}-\mathrm{NMR}\left(\mathrm{C}_{6} \mathrm{D}_{6}\right): \delta=$ 236.3 (d, $J=3.8 \mathrm{~Hz}$, trans-CO), 226.5 (d, $J=11.9 \mathrm{~Hz}$, cis-CO), $219.0(\mathrm{~d}, J=93.4 \mathrm{~Hz}, \mathrm{P}=\mathrm{C}), 150.4(\mathrm{~d}, J=14.0 \mathrm{~Hz}, \mathrm{C}-1), 128.8$ (s, C-2), 127.6 (s, C-3), 126.8 (s, C-4), 95.7 (d, $\left.J=3.5 \mathrm{~Hz}, \mathrm{C}_{5} \mathrm{H}_{5}\right), 1.7$ $\left[\mathrm{d}, J=10.0 \mathrm{~Hz}, \mathrm{Si}\left(\mathrm{CH}_{3}\right)_{3}\right] .-{ }^{\mathrm{t}} \mathrm{H}-\mathrm{NMR}\left(\mathrm{C}_{6} \mathrm{D}_{6}\right): \delta=7.30(\mathrm{~m}, 5 \mathrm{H}$, $\left.\mathrm{C}_{6} \mathrm{H}_{5}\right), 4.80\left(\mathrm{~d}, J=3 \mathrm{~Hz}, 5 \mathrm{H}, \mathrm{C}_{5} \mathrm{H}_{5}\right), 0.33[\mathrm{~d}, J=5.0 \mathrm{~Hz}, 9 \mathrm{H}$, $\left.\mathrm{Si}\left(\mathrm{CH}_{3}\right)_{3}\right]$. - MS, $m / z(\%): 440(4)\left[\mathrm{M}^{+}\right], 412(12)\left[\mathrm{M}^{+}-\mathrm{CO}\right.$, $354(61)\left[\mathrm{M}^{+}-3 \mathrm{CO}\right], 281(15)\left[\mathrm{M}^{+}-3 \mathrm{CO}-\mathrm{SiMe}_{3}\right], 120(5)$
$\left[\mathrm{P}(\mathrm{C}) \mathrm{C}_{6} \mathrm{H}_{5}^{+}\right], 73(100)\left[\mathrm{SiMe}_{3}^{+}\right], 28(23)\left[\mathrm{CO}^{+}\right]$und weitere Bruchstücke. - IR ( $v_{\mathrm{CO}}$, Pentan): $2000 \mathrm{~cm}^{-1}$ st, $1940 \mathrm{st}, 1928$ sst.

$$
\begin{array}{ll}
\mathrm{C}_{18} \mathrm{H}_{19} \mathrm{MoO}_{3} \mathrm{PSi}(440.0) & \text { Ber. C } 49.09 \mathrm{H} 4.37 \\
& \text { Gef. C } 48.96 \mathrm{H} 4.21
\end{array}
$$

Tricarbonyl ( $\eta^{5}$-pentamethylcyclopentadienyl) \{ / phenyl(trimethylsilyl ) methylen]phosphanyl \}molybdän (4b): Eine Lösung von $5 \mathrm{mmol}$ 2b in $50 \mathrm{ml}$ THF wird bei $-78^{\circ} \mathrm{C}$ mit $1.37 \mathrm{~g}(5 \mathrm{mmol}) 1 \mathrm{~b}$ versetzt. Es wird $1 \mathrm{~h}$ bei dieser Temp. gerührt und anschließend innerhalb von $2 \mathrm{~h}$ auf Raumtemp. erwärmt. Das Lösungsmittel wird i. Vak. entfernt, der orangerote Rückstand in $30 \mathrm{ml}$ Pentan aufgenommen und die Lösung filtriert. Kristallisation bei $-25^{\circ} \mathrm{C}$ liefert $4 \mathrm{~b}$ in orangefarbenen Kristallen, Schmp. $86^{\circ} \mathrm{C}$, Ausb. $1.65 \mathrm{~g}(65 \%)$. ${ }^{31} \mathrm{P}-\mathrm{NMR}\left(\mathrm{C}_{6} \mathrm{D}_{6}\right): \delta=471.3 .-{ }^{13} \mathrm{C}-\mathrm{NMR}\left(\mathrm{C}_{6} \mathrm{D}_{6}\right): \delta=237.6(\mathrm{~d}, J$ $=2 \mathrm{~Hz}$, trans-CO), 227.5 (d, $J=12.9 \mathrm{~Hz}$, cis-CO), 217.9 (d, $J=$ $91.1 \mathrm{~Hz}, \mathrm{P}=\mathrm{C}), 150.0(\mathrm{~d}, J=14.6 \mathrm{~Hz}, \mathrm{C}-1), 127.6(\mathrm{~s}, \mathrm{C}-2), 126.8(\mathrm{~s}$, C-3), $125.2(\mathrm{~s}, \mathrm{C}-4), 105.5\left[\mathrm{~s}, \mathrm{C}_{5}\left(\mathrm{CH}_{3}\right)_{5}\right], 10.2\left[\mathrm{~s}, \mathrm{C}_{5}\left(\mathrm{CH}_{3}\right)_{5}\right], 0.5[\mathrm{~d}$, $\left.J=10.1 \mathrm{~Hz}, \mathrm{Si}\left(\mathrm{CH}_{3}\right)_{3}\right] .-{ }^{1} \mathrm{H}-\mathrm{NMR}\left(\mathrm{C}_{6} \mathrm{D}_{6}\right): \delta=6.95(\mathrm{br}, 5 \mathrm{H}$, $\left.\mathrm{C}_{6} \mathrm{H}_{5}\right), 1.40\left[\mathrm{~s}, 15 \mathrm{H}, \mathrm{C}_{5}\left(\mathrm{CH}_{3}\right)_{5}\right], 0.18\left[\mathrm{~d}, J=1.0 \mathrm{~Hz}, 9 \mathrm{H}, \mathrm{Si}\left(\mathrm{CH}_{3}\right)_{3}\right]$. - MS, $m / z(\%): 508(2)\left[\mathrm{M}^{+}\right], 480(9)\left[\mathrm{M}^{+}-\mathrm{CO}\right], 424(100)\left[\mathrm{M}^{+}\right.$ $-3 \mathrm{CO}], 351$ (49) $\left[\mathrm{M}^{+}-3 \mathrm{CO}-\mathrm{SiMe}_{3}\right], 77$ (12) $\left[\mathrm{C}_{6} \mathrm{H}_{5}^{+}\right], 73$ (46) $\left[\mathrm{SiMe}_{3}^{+}\right]$und weitere Bruchstücke. - IR (v $v_{\mathrm{CO}}$, Pentan): 1991 $\mathrm{cm}^{-1}$ sst, $1938 \mathrm{sch}, 1910$ br.

\section{$\mathrm{C}_{23} \mathrm{H}_{29} \mathrm{MoO}_{3}$ PSi (508.2) Ber. C 54.31 H 5.75 Gef. C 54.07 H 5.62}

Tricarbonyl $\left(\eta^{5}\right.$-cyclopentadienyl) \{[phenyl(trimethylsilyl) methylen]phosphanyl \}wolfram (4c): Eine Suspension von $1.47 \mathrm{~g}(4.12$ $\mathrm{mmol}) 2 \mathrm{c}$ in $40 \mathrm{ml}$ Toluol wird bei $-78^{\circ} \mathrm{C}$ mit $1.13 \mathrm{~g}(4.12 \mathrm{mmol})$ 1 b versetzt. Nach Erwärmen auf Raumtemp. wird 2 h gerührt, dann wird abfiltriert, das Lösungsmittel i.Vak. entfernt, der Rückstand in $10 \mathrm{ml}$ Pentan aufgenommen, $4 \mathrm{c}$ bei $-78^{\circ} \mathrm{C}$ ausgefroren und i. Vak. getrocknet. Man erhält $1.30 \mathrm{~g}(63 \%)$ gelboranges Kristallpulver, Schmp. $70^{\circ} \mathrm{C}$. $-{ }^{31} \mathrm{P}-\mathrm{NMR}\left(\mathrm{C}_{3} \mathrm{D}_{6} \mathrm{O}\right): \delta=403.3\left({ }^{1} J_{\mathrm{wP}}=\right.$ $\left.4.4,{ }^{2} J_{\mathrm{PCS} i}=46.9 \mathrm{~Hz}\right) .-{ }^{13} \mathrm{C}-\mathrm{NMR}\left(\mathrm{C}_{6} \mathrm{D}_{6}\right): \delta=224.1\left(\mathrm{~d},{ }^{2} J_{\mathrm{CWP}}=\right.$ $5.0 \mathrm{~Hz}$, trans-CO), $215.7\left(\mathrm{~d},{ }^{2} J_{\mathrm{CWP}}=8.3 \mathrm{~Hz}\right.$, cis-CO), $212.5\left(\mathrm{~d},{ }^{1} J_{\mathrm{CP}}\right.$ $=91.6 \mathrm{~Hz}, \mathrm{P}=\mathrm{C}), 150.0\left(\mathrm{~d},{ }^{2} J_{\mathrm{CCP}}=15.2 \mathrm{~Hz}, \mathrm{C}-1\right), 127.8(\mathrm{~s}, \mathrm{C}-3)$, $126.5\left(\mathrm{~d},{ }^{3} J_{\mathrm{CCCP}}=8.8 \mathrm{~Hz}, \mathrm{C}-2\right), 125.5(\mathrm{~s}, \mathrm{C}-4), 93.2\left(\mathrm{~d},{ }^{2} J_{\mathrm{CWP}}=3.5\right.$ $\left.\mathrm{Hz}, 5 \mathrm{C}, \mathrm{C}_{5} \mathrm{H}_{5}\right), 0.54\left[\mathrm{~d},{ }^{3} \mathrm{~J}_{\mathrm{CSiCP}}=10.1 \mathrm{~Hz}, 3 \mathrm{C}, \mathrm{Si}\left(\mathrm{CH}_{3}\right)_{3}\right] .-{ }^{1} \mathrm{H}-$ $\operatorname{NMR}\left(\mathrm{C}_{6} \mathrm{D}_{6}\right): \delta=7.30-6.97\left(\mathrm{~m}, 5 \mathrm{H}, \mathrm{C}_{6} \mathrm{H}_{5}\right), 4.53\left(\mathrm{~d},{ }^{3} J_{\mathrm{HCWP}}=1.4\right.$ $\left.\mathrm{Hz}, 5 \mathrm{H}, \mathrm{C}_{5} \mathrm{H}_{5}\right), 0.00\left[\mathrm{~d},{ }^{4} \mathrm{~J}_{\mathrm{HCSiCP}}=1.4 \mathrm{~Hz}, 9 \mathrm{H}, \mathrm{Si}\left(\mathrm{CH}_{3}\right)_{3}\right] .-{ }^{29} \mathrm{Si}-$ $\operatorname{NMR}\left(\mathrm{C}_{3} \mathrm{D}_{6} \mathrm{O}\right): \delta=-3.5\left(\mathrm{~d},{ }^{2} J_{\mathrm{SiCP}}=46.9 \mathrm{~Hz}\right) .-$ IR $\left(\mathrm{v}_{\mathrm{CO}}, \mathrm{Me}-\right.$ thylcyclohexan): $2002 \mathrm{~cm}^{-1} \mathrm{~s}$, sh, 1938 vs, 1924 vs. - MS, $m / z(\%)$ : $526(0.47)\left[\mathrm{M}^{+}\right], 498(3.31)\left[\mathrm{M}^{+}-\mathrm{CO}\right], 470(1.02)\left[\mathrm{M}^{+}-2 \mathrm{CO}\right]$, 442 (6.97) $\left[\mathrm{M}^{+}-3 \mathrm{CO}\right], 369(3.15)\left[\mathrm{M}^{+}-3 \mathrm{CO}-\mathrm{SiMe}_{3}\right], 304$ (2.91) $\left[\mathrm{M}^{+}-3 \mathrm{CO}-\mathrm{SiMe}_{3}-\mathrm{C}_{5} \mathrm{H}_{5}\right], 249(3.20)\left[\mathrm{CpW}^{+}\right], 73$ (61.74) $\left[\mathrm{SiMe}_{3}^{+}\right]$und weitere Bruchstücke.

\section{$\mathrm{C}_{18} \mathrm{H}_{19} \mathrm{O}_{3}$ PSiW (526.0) Ber. C 41.08 H 3.64 Gef. C 40.58 H 3.53}

Tricarbonyl ( $\eta^{5}$-pentamethylcyclopentadienyl) \{ [phenyl(trimethylsilyl) methylen] phosphanyl $\}$ wolfram (4d): Analog 4c aus $0.65 \mathrm{~g}(1.58$ mmol) $2 \mathrm{~d}$ und $0.43 \mathrm{~g}(1.58 \mathrm{mmol}) 1 \mathrm{~b}$ in $30 \mathrm{ml}$ Petrolether $\left(30-70^{\circ} \mathrm{C}\right.$ ). $4 \mathrm{~d}$ wird säulenchromatographisch (Säule $1.0 \times 20 \mathrm{~cm}$, $\mathrm{Al}_{2} \mathrm{O}_{3}$ III, Methylcyclohexan/Ether 5:1) von gleichzeitig entstandenem $\mathrm{C}_{5} \mathrm{Me}_{5}(\mathrm{CO})_{3} \mathrm{WBr}$ abgetrennt. Die orangefarbene Fraktion wird aufgefangen und $\mathbf{4 d}$ nach Abdampfen des Solvens i. Vak. als orangefarbenes Kristallpulver erhalten. Ausb. $0.48 \mathrm{~g}(51 \%)$, Schmp. $136-138^{\circ} \mathrm{C}$ (Zers.). - ${ }^{31} \mathrm{P}-\mathrm{NMR}\left(\mathrm{C}_{6} \mathrm{D}\right): \delta=441.0\left({ }^{1} J_{\mathrm{PW}}=9.5 \mathrm{~Hz}\right)$. $-{ }^{13} \mathrm{C}-\mathrm{NMR}\left(\mathrm{C}_{6} \mathrm{D}_{6}\right): \delta=227.4\left(\mathrm{~d},{ }^{2} J_{\mathrm{CWP}}=6.0 \mathrm{~Hz}\right.$, trans-CO), 219.4 $\left(\mathrm{d},{ }^{2} J_{\mathrm{CWP}}=9.3 \mathrm{~Hz}\right.$, cis-CO), $212.8\left(\mathrm{~d},{ }^{1} J_{\mathrm{CP}}=89.0 \mathrm{~Hz}, \mathrm{P}=\mathrm{C}\right), 150.8$ $\left(\mathrm{d},{ }^{2} J_{\mathrm{CCP}}=15.2 \mathrm{~Hz}, \mathrm{C}-1\right), 127.7(\mathrm{~s}, \mathrm{C}-3), 126.6\left(\mathrm{~d},{ }^{3} J_{\mathrm{CCCP}}=9.0 \mathrm{~Hz}\right.$, C-2), $125.3(\mathrm{~s}, \mathrm{C}-4), 104.0\left[\mathrm{~s}, C_{5}\left(\mathrm{CH}_{3}\right)_{5}\right], 10.0\left[\mathrm{~d},{ }^{3} J_{\text {CCWP }}=7.1 \mathrm{~Hz}\right.$, $\left.\mathrm{C}_{5}\left(\mathrm{CH}_{3}\right)_{5}\right], 0.5\left[\mathrm{~d},{ }^{3} J_{\mathrm{CSiCP}}=10.1 \mathrm{~Hz}, \mathrm{Si}\left(\mathrm{CH}_{3}\right)_{3}\right] .-{ }^{1} \mathrm{H}-\mathrm{NMR}\left(\mathrm{C}_{6} \mathrm{D}_{6}\right)$ : 
$\delta=7.35-7.14\left(\mathrm{~m}, 5 \mathrm{H}, \mathrm{C}_{6} \mathrm{H}_{5}\right), 1.66\left[\mathrm{~s}, 15 \mathrm{H}, \mathrm{C}_{5}\left(\mathrm{CH}_{3}\right)_{5}\right], 0.35[\mathrm{~d}$, $\left.{ }^{4} J_{\mathrm{HCSiCP}}=1.2 \mathrm{~Hz}, 9 \mathrm{H}, \mathrm{Si}\left(\mathrm{CH}_{3}\right)_{3}\right] .-{ }^{29} \mathrm{Si}-\mathrm{NMR}\left(\mathrm{C}_{6} \mathrm{D}_{6}\right): \delta=-3.7$ $\left(\mathrm{d},{ }^{2} J_{\mathrm{SiCP}}=43.2 \mathrm{~Hz}\right) .-\mathrm{IR}\left(\mathrm{v}_{\mathrm{CO}}\right.$, Petrolether $\left.40-60^{\circ} \mathrm{C}\right): 1996 \mathrm{~cm}^{-1}$ sst, 1925 s, 1914 sst.

\section{$\mathrm{C}_{23} \mathrm{H}_{29} \mathrm{O}_{3} \mathrm{PSiW}$ (596.1) Ber. C $46.32 \mathrm{H} 4.90$ Gef. C 46.05 H 5.14}

Dicarbonyl $\left(\eta^{5}\right.$-cyclopentadienyl) \{[phenyl(trimethylsilyl)methylen]phosphanyl \}eisen (4e): $3.2 \mathrm{mmol} \mathbf{2 e}$, gelöst in $40 \mathrm{ml}$ THF werden bei $-78^{\circ} \mathrm{C}$ mit $0.87 \mathrm{~g}(3.2 \mathrm{mmol}) 1 \mathrm{~b}$ versetzt. Es wird $1 \mathrm{~h}$ bei dieser und weitere $2 \mathrm{~h}$ bei Raumtemp. gerührt, zur Trockene eingeengt und der Rückstand in $50 \mathrm{ml}$ Pentan aufgenommen. Die Kristallisation aus Pentan/Diethylether $(1: 1)$ bei $0^{\circ} \mathrm{C}$ liefert $4 \mathrm{e}$ in orangefarbenen Plättchen. Ausb. $0.81 \mathrm{~g}(68 \%)$, Schmp. $74^{\circ} \mathrm{C}$. ${ }^{31}$ P-NMR $\left(\mathrm{C}_{6} \mathrm{D}_{6}\right): \delta=476.3 .-{ }^{13} \mathrm{C}-\mathrm{NMR}\left(\mathrm{C}_{6} \mathrm{D}_{6}\right): \delta=217.2(\mathrm{~d}$, $J=98.3 \mathrm{~Hz}, \mathrm{P}=\mathrm{C}$ ), 214.9 (s, CO), 151.9 (d, $J=12.9 \mathrm{~Hz}, \mathrm{C}-1), 130.1$ (s, C-2), 128.9 (s, C-3), 125.9 (s, C-4), 86.8 (d, $\left.J=2.8 \mathrm{~Hz}, \mathrm{C}_{5} \mathrm{H}_{5}\right), 1.5$ $\left[\mathrm{d}, J=9.8 \mathrm{~Hz}, \mathrm{Si}\left(\mathrm{CH}_{3}\right)_{3}\right] .-{ }^{1} \mathrm{H}-\mathrm{NMR}\left(\mathrm{C}_{6} \mathrm{D}_{6}\right): \delta=7.25(\mathrm{br}, 5 \mathrm{H}$, $\left.\mathrm{C}_{6} \mathrm{H}_{5}\right), 4.05\left(\mathrm{~d}, J=2.5 \mathrm{~Hz}, 5 \mathrm{H}, \mathrm{C}_{5} \mathrm{H}_{5}\right), 0.40\left[\mathrm{~d}, 3.2 \mathrm{~Hz}, 9 \mathrm{H}, \mathrm{Si}\left(\mathrm{CH}_{3}\right)_{3}\right]$. - MS, $m / z(\%): 370(1)\left[\mathrm{M}^{+}\right], 342(1)\left[\mathrm{M}^{+}-\mathrm{CO}\right], 314$ (9) $\left[\mathrm{M}^{+}\right.$ $-2 \mathrm{CO}], 241(15)\left[\mathrm{M}^{+}-2 \mathrm{CO}-\mathrm{SiMe}_{3}\right], 121(25)\left[\mathrm{C}_{5} \mathrm{H}_{5} \mathrm{Fe}^{+}\right]$, $73(100)\left[\mathrm{SiMe}_{3}^{+}\right], 28(5)\left[\mathrm{CO}^{+}\right]$und weitere Bruchstücke. - IR ( $v_{\text {CO }}$, Pentan): $2004 \mathrm{~cm}^{-1}$ s, 1960 vs.

$$
\begin{array}{ll}
\mathrm{C}_{17} \mathrm{H}_{19} \mathrm{FeO}_{2} \mathrm{PSi} \text { (370.0) } & \text { Ber. C } 55.13 \text { H } 5.18 \\
& \text { Gef. C } 55.26 \text { H } 5.23
\end{array}
$$

Dicarbonyl $\left(\eta^{5}\right.$-pentamethylcyclopentadienyl) \{ [phenyl (trimethylsilyl)methylen]phosphanyl \}eisen (4f): Eine auf $-40^{\circ} \mathrm{C}$ gekühlte Lösung von $4 \mathrm{mmol} 2 \mathrm{f}$ in einem Gemisch aus $25 \mathrm{ml}$ Petrolether (Siedebereich $\left.40-60^{\circ} \mathrm{C}\right)$ und $2 \mathrm{ml}$ THF wird mit $1.10 \mathrm{~g}(4 \mathrm{mmol}) \mathbf{1 b}$ versetzt. Nachdem auf Raumtemp. erwärmt wurde, wird weitere $12 \mathrm{~h}$ gerührt, zur Trockene eingeengt und der Rückstand dreimal mit je $15 \mathrm{ml}$ Petrolether extrahiert. Die Kristallisation bei $-35^{\circ} \mathrm{C}$ liefert $\mathbf{4 f}$ in orangefarbenen Plättchen, Ausb. $1.19 \mathrm{~g} \mathrm{(68 \% )}$, Schmp. $85^{\circ} \mathrm{C} .-{ }^{31} \mathrm{P}-\mathrm{NMR}\left(\mathrm{C}_{6} \mathrm{D}_{6}\right): \delta=520.0 .-{ }^{13} \mathrm{C}-\mathrm{NMR}\left(\mathrm{C}_{6} \mathrm{D}_{6}\right): \delta=$ 216.0 (s, CO), $211.1(\mathrm{~d}, J=87.4 \mathrm{~Hz}, \mathrm{P}=\mathrm{C}), 151.6(\mathrm{~d}, J=13.9 \mathrm{~Hz}$, C-1), 130.4 (s, C-2), 127.9 (s, C-3), 124.9 (s, C-4), 97.2 [d, $J=6.4$ $\left.\mathrm{Hz}, C_{5}\left(\mathrm{CH}_{3}\right)_{5}\right], 9.0\left[\mathrm{~d}, J=8.5 \mathrm{~Hz}, \mathrm{C}_{5}\left(\mathrm{CH}_{3}\right)_{5}\right], 1.3[\mathrm{~d}, J=9.7 \mathrm{~Hz}$, $\left.\mathrm{Si}\left(\mathrm{CH}_{3}\right)_{3}\right] .-{ }^{1} \mathrm{H}-\mathrm{NMR}\left(\mathrm{C}_{6} \mathrm{D}_{6}\right): \delta=7.23-6.98\left(\mathrm{~m}, 5 \mathrm{H}, \mathrm{C}_{6} \mathrm{H}_{5}\right), 1.3$ $\left[\mathrm{s}, 15 \mathrm{H}, \mathrm{C}_{5}\left(\mathrm{CH}_{3}\right)_{5}\right], 0.34\left[\mathrm{~d}, J=1.0 \mathrm{~Hz}, 9 \mathrm{H}, \mathrm{Si}\left(\mathrm{CH}_{3}\right)_{3}\right] .-\mathrm{MS}$, $m / z(\%): 440(1)\left[\mathrm{M}^{+}\right], 384(8)\left[\mathrm{M}^{+}-2 \mathrm{CO}\right], 311(53)\left[\mathrm{M}^{+}-\right.$ $\left.2 \mathrm{CO}-\mathrm{SiMe}_{3}\right], 190$ (13) $\left[\mathrm{C}_{5} \mathrm{Me}_{5} \mathrm{Fe}^{+}\right], 135$ (19) $\left[\mathrm{C}_{5} \mathrm{Me}_{5}^{+}\right], 134$ (9) $\left[\mathrm{C}_{5} \mathrm{Me}_{5}^{+}-\mathrm{H}\right], 119(28)\left[\mathrm{C}_{5} \mathrm{Me}_{5}^{+}-\mathrm{CH}_{4}\right], 77(15)\left[\mathrm{C}_{6} \mathrm{H}_{5}^{+}\right], 73(100)$ $\left[\mathrm{SiMe}_{3}^{+}\right]$und weitere Bruchstücke. - IR ( $v_{\mathrm{CO}}$, Pentan): $1995 \mathrm{~cm}^{-1}$ st, 1948 st.

\section{$\mathrm{C}_{22} \mathrm{H}_{29} \mathrm{FeO}_{2} \mathrm{PSi}$ (440.1) Ber. C 59.98 H 6.64 \\ Gef. C 59.77 H 6.45}

$\left(\eta^{5}\right.$-Pentamethylcyclopentadienyl $)\{[$ phenyl(trimethylsilyl) methylen]phosphanyl\}(triethylphosphan)nickel (4g): Eine Lösung von $0.605 \mathrm{~g} 7 \mathrm{a}(1.5 \mathrm{mmol})$ in Toluol wird auf $0^{\circ} \mathrm{C}$ gekühlt und unter Rühren zügig mit $0.493 \mathrm{~g} \mathbf{5 b}(1.5 \mathrm{mmol})$ versetzt, wobei fast augenblicklich ein Farbumschlag zu erkennen ist. Nach beendeter Zugabe wird die Reaktionslösung noch $2 \mathrm{~h}$ bei Raumtemp. gerührt. Anschließendes Entfernen des Lösungsmittels i. Vak. und Umkristallisieren aus Benzol/Pentan liefert amorphes $4 \mathrm{~g}$, Ausb. $0.55 \mathrm{~g}(73 \%)$, Schmp. $73-74^{\circ}$ C. $-{ }^{31}$ P-NMR $\left(\mathrm{C}_{6} \mathrm{D}_{6}\right): \delta=619.3(\mathrm{~d}, J=14.1 \mathrm{~Hz}$, $\mathrm{P}=\mathrm{C}), 25.7\left(\mathrm{~d}, J=14.1 \mathrm{~Hz}, \mathrm{PR}_{3}\right) .-{ }^{13} \mathrm{C}-\mathrm{NMR}\left(\mathrm{C}_{6} \mathrm{D}_{6}\right): \delta=202.6$ (d, breit, $J=94.7 \mathrm{~Hz}, \mathrm{P}=\mathrm{C}$ ), 151.7 (m, C-1), 125.3-124.7 (m, C-2 bis C-4), $99.6\left[\mathrm{~s}, C_{5}\left(\mathrm{CH}_{3}\right)_{5}\right], 16.4(\mathrm{~d}, J=27.2 \mathrm{~Hz}, \mathrm{PCC}), 10.4[\mathrm{~s}$, $\left.\mathrm{C}_{5}\left(\mathrm{CH}_{3}\right)_{5}\right], 7.5(\mathrm{~s}, \mathrm{PCC}), 2.3\left[\mathrm{~d}, 11 \mathrm{~Hz}, \mathrm{Si}\left(\mathrm{CH}_{3}\right)_{3}\right] .-{ }^{1} \mathrm{H}-\mathrm{NMR}\left(\mathrm{C}_{6} \mathrm{D}_{6}\right)$ : $\delta=7.24\left(\mathrm{~b}, 5 \mathrm{H}, \mathrm{C}_{6} \mathrm{H}_{5}\right), 1.77\left[\mathrm{~d}, J=0.9 \mathrm{~Hz}, 15 \mathrm{H}, \mathrm{C}_{5}\left(\mathrm{CH}_{3}\right)_{5}\right], 0.99$ $\left(\mathrm{s}, 6 \mathrm{H}, \mathrm{PCH}_{2}\right), 0.74\left(\mathrm{~s}, 9 \mathrm{H}, \mathrm{PCCH}_{3}\right), 0.47[\mathrm{~d}, J=1.4 \mathrm{~Hz}, 9 \mathrm{H}$, $\left.\mathrm{Si}\left(\mathrm{CH}_{3}\right)_{3}\right]$. - MS (FAB/mNBA), $m / z(\%): 658.5(38.2)[\mathrm{M}+m \mathrm{NBA}$
$\left.+\mathrm{H}^{+}\right], 523.4(20)\left[\mathrm{M}+\mathrm{H}_{2} \mathrm{O}+\mathrm{H}^{+}\right], 311.2(100)\left[\mathrm{M}^{+}-\right.$ $\left.\mathrm{P}=\mathrm{C}\left(\mathrm{SiMe}_{3}\right)(\mathrm{Ph})\right]$ und weitere Bruchstücke.

$$
\begin{array}{ll}
\mathrm{C}_{26} \mathrm{H}_{44} \mathrm{NiP}_{2} \mathrm{Si} \text { (504.2) } & \text { Ber. C } 61.88 \text { H } 8.79 \\
& \text { Gef. C } 62.11 \text { H } 8.59
\end{array}
$$

$\left(\eta^{5}\right.$-Pentamethylcyclopentadienyl) \{[phenyl(trimethylsilyl)methylen]phosphanyl\}(tributylphosphan) nickel (4h): $0.493 \mathrm{~g}$ 5b werden bei $-10^{\circ} \mathrm{C}$ in eine Lösung von $0.857 \mathrm{~g} 7 \mathrm{~b}$ in $6 \mathrm{ml}$ Benzol/Pentan (1:1) getropft. Nach 30 min wird auf Raumtemp. erwärmt und $1 \mathrm{~h}$ nachgerührt. Das Lösungsmittel wird i.Vak. entfernt und das schwarze Rohprodukt aus Toluol umkristallisiert. Man erhält $\mathbf{4 h}$ in kleinen braunschwarzen Nadeln. Ausb. $0.66 \mathrm{~g}(75 \%)$, Schmp. $77^{\circ} \mathrm{C}$. $-{ }^{31} \mathrm{P}-\mathrm{NMR}\left(\mathrm{C}_{6} \mathrm{D}_{6}\right): \delta=620.1(\mathrm{~d}, J=14.8 \mathrm{~Hz}, \mathrm{P}=\mathrm{C}), 16.5$ (d, $\left.J=14.8 \mathrm{~Hz}, \mathrm{PR}_{3}\right) .-{ }^{13} \mathrm{C}-\mathrm{NMR}\left(\mathrm{C}_{6} \mathrm{D}_{6}\right): \delta=201.6(\mathrm{dd}, J=$ 93.7 und $4 \mathrm{~Hz}, \mathrm{P}=\mathrm{C}$ ), 151.5 (d, $J=12.4 \mathrm{~Hz}, \mathrm{C}-1), 127.5$ (s, C-3), 126.6 (s, C-2), 124.9 (s, C-4), 99.7 [d, $J=1.4 \mathrm{~Hz}, C_{5}\left(\mathrm{CH}_{3}\right)_{5}$ ], 28.8 (s, PC), 24.7 (dd, $J=25.6$ und $8.8 \mathrm{~Hz}, \mathrm{PCC}), 24.5(\mathrm{~d}, J=11.9 \mathrm{~Hz}$, PCCC), 14.0 (s, PCCCC), $10.2\left[\mathrm{~d}, J=5.1 \mathrm{~Hz}, \mathrm{C}_{5}\left(\mathrm{CH}_{3}\right)_{5}\right], 2.35$ [d, $\left.J=11 \mathrm{~Hz}, \mathrm{Si}\left(\mathrm{CH}_{3}\right)_{3}\right] .-{ }^{\prime} \mathrm{H}-\mathrm{NMR}\left(\mathrm{C}_{6} \mathrm{D}_{6}\right): \delta=7.24$ (br, $\left.5 \mathrm{H}, \mathrm{C}_{5} \mathrm{H}_{5}\right)$, $1.8\left[\mathrm{~d}, J=0.9 \mathrm{~Hz}, 15 \mathrm{H}, \mathrm{C}_{5}\left(\mathrm{CH}_{3}\right)_{5}\right], 1.38\left(\mathrm{br}, 6 \mathrm{H}, \mathrm{PCH}_{2}\right), 1.1(\mathrm{br}$, $\left.12 \mathrm{H}, \mathrm{PCCH} \mathrm{CH}_{2} \mathrm{C}\right), 0.9\left(\mathrm{~s}, 9 \mathrm{H}, \mathrm{PCCCCH}_{3}\right), 0.47[\mathrm{~d}, J=1.2 \mathrm{~Hz}$, $\left.9 \mathrm{H}, \mathrm{Si}\left(\mathrm{CH}_{3}\right)_{3}\right]$. - MS (FAB $\left./ m \mathrm{NBA}\right), m / z(\%): 742.6$ (18.2) $[\mathrm{M}+$ $\left.m \mathrm{NBA}+\mathrm{H}^{+}\right], 607.7(8.2)\left[\mathrm{M}+\mathrm{H}_{2} \mathrm{O}+\mathrm{H}^{+}\right], 395.4$ (70.9) $\left[\mathrm{M}^{+}\right.$ - $\left.\mathrm{P}=\mathrm{C}\left(\mathrm{SiMe}_{3}\right)(\mathrm{Ph})\right]$ und weitere Bruchstücke.

$$
\begin{array}{lll}
\mathrm{C}_{32} \mathrm{H}_{56} \mathrm{NiP}_{2} \mathrm{Si} \text { (588.3) } & \text { Ber. C } 65.27 \text { H } 9.59 \\
& \text { Gef. C } 65.38 \text { H } 9.39
\end{array}
$$

$\left(\eta^{5}\right.$-Pentamethylcyclopentadienyl) \{[phenyl(trimethylsilyl)methylen]phosphanyl\}(triphenylphosphan)nickel (4i): Zu einer Lösung von $1.037 \mathrm{~g} 7 \mathrm{c}(1.5 \mathrm{mmol})$ in $7 \mathrm{ml}$ Benzol/Pentan (1:1) werden bei $-10^{\circ} \mathrm{C} 0.493 \mathrm{~g} \mathrm{5b}$ gegeben. Man läßt $30 \mathrm{~min}$ in der Kälte nachrühren, wobei sich die Lösung dunkelbraun färbt. Nach Erwärmen auf Raumtemp. wird noch $1 \mathrm{~h}$ gerührt, anschließend i. Vak. bis zur Trockene eingeengt. Der braunschwarze Rückstand wird mit wenig kaltem Toluol gewaschen, Ausb. $0.65 \mathrm{~g}(67 \%)$, Schmp. $80^{\circ} \mathrm{C}$. ${ }^{31} \mathrm{P}-\mathrm{NMR}\left(\mathrm{C}_{6} \mathrm{D}_{6}\right): \delta=602.3$ (d, $\left.J=27.9 \mathrm{~Hz}, \mathrm{P}=\mathrm{C}\right), 43.5$ (d, $J=$ $\left.27.9 \mathrm{~Hz}, \mathrm{PR}_{3}\right) .-{ }^{13} \mathrm{C}-\mathrm{NMR}\left(\mathrm{C}_{6} \mathrm{D}_{6}\right): \delta=198.5$ (dd, $J=93.0$ und $5.9 \mathrm{~Hz}, \mathrm{P}=\mathrm{C}), 149.9$ (d, $J=13.1 \mathrm{~Hz}, \mathrm{C}-1), 137.9-121.2(\mathrm{~m}, \mathrm{C}-2$ bis C-4), $100.7\left[\mathrm{~s}, \mathrm{C}_{5}\left(\mathrm{CH}_{3}\right)_{5}\right], 10.0\left[\mathrm{~d}, J=3.6 \mathrm{~Hz}, \mathrm{C}_{5}\left(\mathrm{CH}_{3}\right)_{5}\right], 2.0[\mathrm{~d}$, $\left.J=11 \mathrm{~Hz}, \mathrm{Si}\left(\mathrm{CH}_{3}\right)_{3}\right] .-{ }^{1} \mathrm{H}-\mathrm{NMR}\left(\mathrm{C}_{6} \mathrm{D}_{6}\right): \delta=7.4$ und $7.3(\mathrm{~m}$, $\left.20 \mathrm{H}, \mathrm{C}_{6} \mathrm{H}_{5}\right), 1.48\left[\mathrm{~d}, J=1 \mathrm{~Hz}, 15 \mathrm{H}, \mathrm{C}_{5}\left(\mathrm{CH}_{3}\right)_{5}\right], 0.31[\mathrm{~d}, J=1.7$ $\left.\mathrm{Hz}, 9 \mathrm{H}, \mathrm{Si}\left(\mathrm{CH}_{3}\right)_{3}\right]$. - MS (FAB $\left./ m \mathrm{NBA}\right), m / z(\%): 802.6$ (18.2) [M $\left.+m \mathrm{NBA}+\mathrm{H}^{+}\right], 667.5(20)\left[\mathrm{M}+\mathrm{H}_{2} \mathrm{O}+\mathrm{H}^{+}\right], 649.5(61.8)[\mathrm{M}$ $\left.+\mathrm{H}^{+}\right], 455.4(100)\left[\mathrm{M}^{+}-\mathrm{P}=\mathrm{C}\left(\mathrm{SiMe}_{3}\right)(\mathrm{Ph})\right]$ und weitere Bruchstücke.

$$
\begin{array}{ll}
\mathrm{C}_{38} \mathrm{H}_{44} \mathrm{NiP}_{2} \mathrm{Si} \text { (648.2) } & \text { Ber. C } 70.35 \text { H } 6.84 \\
& \text { Gef. C } 69.83 \text { H } 7.21
\end{array}
$$

\{[Bis(trimethylsilyl)methylen](pentamethylcyclopentadienyl)phosphan\}tetracarbonyleisen (6a) und Tetracarbonyl \{(pentamethylcyclopentadienyl) [phenyl(trimethylsilyl) methylen]phosphan\}eisen (6b): $3.64 \mathrm{~g} \mathrm{(10} \mathrm{mmol)} \mathrm{Fe}_{2}(\mathrm{CO})_{9}$ werden mit einer Lösung von 10 mmol $\mathbf{5 a}$ bzw. 5b in $35 \mathrm{ml}$ Hexan versetzt und $3 \mathrm{~d}$ bei Raumtemp. gerührt. Die tiefrote Lösung wird filtriert, flüchtige Bestandteile werden i.Vak. entfernt, und das zurückbleibende rote Öl wird in $20 \mathrm{ml}$ Toluol/Acetonitril (2:3) aufgenommen. Kristallisation bei $-35^{\circ} \mathrm{C}$ liefert $(41 \%) 6 \mathrm{a}$, säulenchromatographische Aufarbeitung $(10 \times 1 \mathrm{~cm}$, Florisil, Toluol) und Kristallisation aus Pentan bei $-35^{\circ} \mathrm{C}$ liefert $3.90 \mathrm{~g}(85 \%) 6 \mathrm{~b}$ als tiefrote Kristalle.

6a: Schmp. $79^{\circ} \mathrm{C}$ (Zers.). - ${ }^{31} \mathrm{P}-\mathrm{NMR}\left(\mathrm{C}_{6} \mathrm{D}_{6}\right): \delta=371.5 .-{ }^{13} \mathrm{C}$ NMR $\left(\mathrm{C}_{6} \mathrm{D}_{6}\right): \delta=216.1$ (d, $\left.J=15.0 \mathrm{~Hz}, \mathrm{CO}\right), 187.8$ (d, $J=24.5$ $\mathrm{Hz}, \mathrm{P}=\mathrm{C}), 142.8(\mathrm{br})$ und $136.5(\mathrm{br})\left[\mathrm{C}_{5}\left(\mathrm{CH}_{3}\right)_{5}\right], 11.8\left[\mathrm{br}, \mathrm{C}_{5}\left(\mathrm{CH}_{3}\right)_{5}\right]$, $5.1(\mathrm{~d}, J=4.8 \mathrm{~Hz})$ und $4.8(\mathrm{~d}, J=8.2 \mathrm{~Hz})\left[\mathrm{Si}\left(\mathrm{CH}_{3}\right)_{3}\right] .-{ }^{1} \mathrm{H}-\mathrm{NMR}$ $\left(\mathrm{C}_{6} \mathrm{D}_{6}\right): \delta=1.83\left[\mathrm{br}, 15 \mathrm{H}, \mathrm{C}_{5}\left(\mathrm{CH}_{3}\right)_{5}\right], 0.38(\mathrm{~s}, 9 \mathrm{H})$ und $0.33(\mathrm{~s}, 9 \mathrm{H})$ [Si( $\left.\left.\mathrm{CH}_{3}\right)_{3}\right] .-\mathrm{MS}, m / z(\%): 492(0.4)\left[\mathrm{M}^{+}\right], 477(0.4)\left[\mathrm{M}^{+}-\mathrm{Me}\right]$, 
$436(3)\left[\mathrm{M}^{+}-2 \mathrm{CO}\right], 408(27)\left[\mathrm{M}^{+}-3 \mathrm{CO}\right], 380(76)\left[\mathrm{M}^{+}-\right.$ $4 \mathrm{CO}], 273(10)\left[\mathrm{M}^{+}-3 \mathrm{CO}-\mathrm{Me}_{5} \mathrm{C}_{5}\right], 245(16)\left[\mathrm{M}^{+}-4 \mathrm{CO}\right.$ $\left.-\mathrm{Me}_{5} \mathrm{C}_{5}\right], 73(100)\left[\mathrm{Me}_{3} \mathrm{Si}^{+}\right]$. - IR ( $v_{\mathrm{co}}$, Hexan): $2053 \mathrm{~cm}^{-1} \mathrm{~m}$, 1976 sst, 1960 st.

\section{$\mathrm{C}_{21} \mathrm{H}_{33} \mathrm{FePO}_{4} \mathrm{Si}_{2}(492.1) \quad$ Ber. C $51.22 \mathrm{H} 6.75$} Gef. C 51.06 H 6.97

6b: Schmp. $99^{\circ} \mathrm{C}$. $-{ }^{31} \mathrm{P}-\mathrm{NMR}\left(\mathrm{C}_{6} \mathrm{D}_{6}\right): \delta=276.9 .-{ }^{13} \mathrm{C}-\mathrm{NMR}$ $\left(\mathrm{C}_{6} \mathrm{D}_{6}\right): \delta=218.6(\mathrm{~d}, J=16.0 \mathrm{~Hz}, \mathrm{CO}), 198.2(\mathrm{~d}, J=11.7 \mathrm{~Hz}$, $\mathrm{P}=\mathrm{C}), 145.3(\mathrm{~d}, J=19.0 \mathrm{~Hz}, \mathrm{C}-1), 138.5\left[\mathrm{~d}, J=10.5 \mathrm{~Hz}, C_{5}\left(\mathrm{CH}_{3}\right)_{5}\right]$, 128.4 (d, $J=3.7 \mathrm{~Hz}, \mathrm{C}-2$ ), 127.6 (s, C-3), 126.1 (s, C-4), 12.7 [br, $\left.\mathrm{C}_{5}\left(\mathrm{CH}_{3}\right)_{5}\right], 1.3\left[\mathrm{~s}, \mathrm{Si}\left(\mathrm{CH}_{3}\right)_{3}\right] .-{ }^{1} \mathrm{H}-\mathrm{NMR}\left(\mathrm{C}_{6} \mathrm{D}_{6}\right): \delta=7.0-6.85(\mathrm{~m}$, $\left.5 \mathrm{H}, \mathrm{C}_{6} \mathrm{H}_{5}\right), 1.6\left[\mathrm{br}, 15 \mathrm{H}, \mathrm{C}_{5}\left(\mathrm{CH}_{3}\right)_{5}\right], 0.21[\mathrm{~d}, J=2.0 \mathrm{~Hz}, 9 \mathrm{H}$, $\left.\mathrm{Si}\left(\mathrm{CH}_{3}\right)_{3}\right]$. - MS, $m / z(\%): 440(1)\left[\mathrm{M}^{+}-2 \mathrm{CO}\right], 328(5)\left[\mathrm{M}^{+}-\right.$ $\left.\mathrm{C}_{5} \mathrm{Me}_{5}\right], 193(26)\left[\mathrm{M}^{+}-\mathrm{C}_{5} \mathrm{Me}_{5}-\mathrm{Fe}(\mathrm{CO})_{4}\right], 135(12)\left[\mathrm{C}_{5} \mathrm{Me}_{5}^{+}\right]$, 134 (15) $\left[\mathrm{C}_{5} \mathrm{Me}_{5}^{+}-\mathrm{H}\right], 119$ (19) $\left[\mathrm{C}_{5} \mathrm{Me}_{5}^{+}-\mathrm{CH}_{4}\right], 77$ (2) $\left[\mathrm{C}_{6} \mathrm{H}_{5}^{+}\right]$, $73(100)\left[\mathrm{SiMe}_{3}^{+}\right]$und weitere Bruchstücke. - IR ( $v_{\mathrm{CO}}$, Pentan): $2040 \mathrm{~cm}^{-1}$ st, $2015 \mathrm{w}, 1970$ sst, 1950 st.

$$
\begin{array}{ll}
\mathrm{C}_{24} \mathrm{H}_{29} \mathrm{FeO}_{4} \mathrm{PSi} \text { (496.1) } & \text { Ber. C } 58.05 \text { H } 5.89 \\
& \text { Gef. C } 56.97 \text { H } 6.07
\end{array}
$$

$\left\{\eta^{2}-[\right.$ Bis (trimethylsilyl) methylen](pentamethylcyclopentadienyl)phosphan $\}$ bis(triethylphosphan)nickel (8) und $\left\{\eta^{2}-(\right.$ Pentamethylcyclopentadienyl) [phenyl(trimethylsilyl)methylen]phosphan\}bis(triethylphosphan) nickel (9a): $0.605 \mathrm{~g} \mathrm{(1.5} \mathrm{mmol)} \mathrm{(Cycloocta-}$ dien)bis(triethylphosphan)nickel in Toluol werden bei $0^{\circ} \mathrm{C}$ unter Rühren mit $0.483 \mathrm{~g}$ ( $1.5 \mathrm{mmol}) 5 \mathrm{a}$ bzw. $0.493 \mathrm{~g}(1.5 \mathrm{mmol}) 5 \mathrm{~b}$ versetzt. Nach beendeter Zugabe wird auf Raumtemp. erwärmt und sofort ein ${ }^{31} \mathrm{P}-\mathrm{NMR}-\mathrm{Sp}$ ektrum aufgenommen.

8: ${ }^{31} \mathrm{P}-\mathrm{NMR}\left(\mathrm{C}_{6} \mathrm{D}_{6}\right): \delta=82.8(\mathrm{~d}$, breit, $J=22 \mathrm{~Hz}, \mathrm{P}=\mathrm{C}), 17.3$ (s, breit, $\mathrm{P}_{\mathrm{a}} \mathrm{Et}_{3}$ ), 10.5 (d, breit, $J=22 \mathrm{~Hz}, \mathrm{P}_{\mathrm{b}} \mathrm{Et}_{3}$ ).

9a: ${ }^{31} \mathrm{P}-\mathrm{NMR}\left(\mathrm{C}_{6} \mathrm{D}_{6}\right): \delta=45.3(\mathrm{t}, J=20.8 \mathrm{~Hz}, \mathrm{P}=\mathrm{C}), 6.7(\mathrm{dd}$, $J=26.9$ und $\left.20.8 \mathrm{~Hz}, \mathrm{P}_{\mathrm{a}} \mathrm{Et}_{3}\right), 3.6\left(\mathrm{dd}, J=26.9\right.$ und $\left.20.8 \mathrm{~Hz}, \mathrm{P}_{\mathrm{b}} \mathrm{Et}_{3}\right)$.

$\left\{\eta^{2}-(\right.$ Pentamethylcyclopentadienyl) [phenyl(trimethylsilyl)methylen]phosphan bis(tributylphosphan)nickel $(9 \mathrm{~b})$ : Eine Lösung von $0.857 \mathrm{~g}(81.5 \mathrm{mmol}) 7 \mathrm{~b}$ in $6 \mathrm{ml}$ Benzol/Pentan $(1: 1)$ wird auf $-10^{\circ} \mathrm{C}$ gekühlt. Nach Zugabe einer äquimolaren Menge 5b wird auf Raumtemp. erwärmt und sofort ein ${ }^{31} \mathrm{P}-\mathrm{NMR}$-Spektrum aufgenommen. - ${ }^{31} \mathrm{P}-\mathrm{NMR}\left(\mathrm{C}_{6} \mathrm{D}_{6}\right): \delta=43.7(\mathrm{t}, J=20.9 \mathrm{~Hz}, \mathrm{P}=\mathrm{C}), 0.8$ (dd, $J=29.1$ und $\left.20.9 \mathrm{~Hz}, \mathrm{P}_{\mathrm{a}} \mathrm{Bu}_{3}\right),-2.2(\mathrm{dd}, J=29.1$ und $20.9 \mathrm{~Hz}$, $\mathrm{P}_{\mathrm{b}} \mathrm{Bu}_{3}$ ).

$\left\{\left[\right.\right.$ Bis(trimethylsilyl) methylen]phoshan \}tricarbonyl ( $\eta^{5}$-cyclopentadienyl) wolfram-trifluormethansulfonat (10a): $\mathrm{Zu}$ einer Lösung von

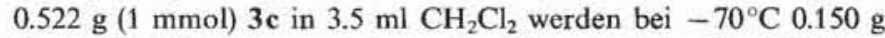
(1 mmol). Triflatsäure gegeben. Die Lösung wird auf $-20^{\circ} \mathrm{C}$ erwärmt, auf $2 \mathrm{ml}$ eingeengt und mit $4 \mathrm{ml}$ Hexan versetzt. Der ausgefallene Niederschlag wird abfiltriert, mehrmals mit Hexan gewaschen und i.Vak. getrocknet. Man erhält $0.30 \mathrm{~g}(45 \%) 10 \mathrm{a}$ als braungelbes Pulver vom Schmp. $94-95^{\circ} \mathrm{C}$ (Zers.). - ${ }^{31} \mathrm{P}-\mathrm{NMR}$ $\left(\mathrm{CD}_{2} \mathrm{Cl}_{2}\right): \delta=187.2\left(J_{\mathrm{WP}}=202 \mathrm{~Hz}\right) .-{ }^{1} \mathrm{H}-\mathrm{NMR}\left(\mathrm{CD}_{2} \mathrm{Cl}_{2}\right): \delta=$ $8.91\left(\mathrm{~d}, J=397, J_{\mathrm{wH}}=15 \mathrm{~Hz}, 1 \mathrm{H}, \mathrm{PH}\right), 6.16(\mathrm{~d}, J=1.3 \mathrm{~Hz}, 5 \mathrm{H}$, $\left.\mathrm{C}_{5} \mathrm{H}_{5}\right), 0.34\left[\mathrm{~s}, 18 \mathrm{H}, \mathrm{Si}\left(\mathrm{CH}_{3}\right)_{3}\right] .-\mathrm{IR}\left(\mathrm{v}_{\mathrm{CO}}, \mathrm{CD}_{2} \mathrm{Cl}_{2}\right): 2058 \mathrm{~cm}^{-1} \mathrm{st}$, 1988 st, 1960 st, br.

\section{$\mathrm{C}_{16} \mathrm{H}_{24} \mathrm{~F}_{3} \mathrm{O}_{6} \mathrm{PSSi}_{2} \mathrm{~W}(672.0)$ Ber. C 28.58 H 3.60 Gef. C 27.26 H 3.77}

$\left\{\left[\right.\right.$ Bis(trimethylsilyl) methylen]methylphosphan \}tricarbonyl $\left(\eta^{5}\right.$ cyclopentadienyl)wolfram-trifluormethansulfonat $(\mathbf{1 0 b})$ : Eine Lö-

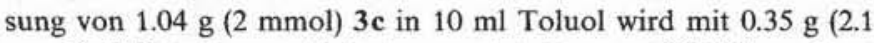
mmol) Triflatsäure-methylester versetzt, kurz auf $30^{\circ} \mathrm{C}$ erwärmt und $48 \mathrm{~h}$ bei Raumtemp. stehengelassen. Der ausgefallene hellgelbe Niederschlag wird abfiltriert, mit Toluol und Hexan gewaschen und i. Vak. getrocknet. Man erhält $1.10 \mathrm{~g} 10 \mathrm{~b}(80 \%)$, Schmp. $89-90^{\circ} \mathrm{C}$ (Zers.). $-{ }^{31}$ P-NMR $\left(\mathrm{CD}_{2} \mathrm{Cl}_{2}\right): \delta=230.8\left(J_{\mathrm{WP}}=198 \mathrm{~Hz}\right) .-{ }^{1} \mathrm{H}-$
$\operatorname{NMR}\left(\mathrm{CD}_{2} \mathrm{Cl}_{2}\right): \delta=6.17\left(\mathrm{~d}, J=0.3 \mathrm{~Hz}, 5 \mathrm{H}, \mathrm{C}_{5} \mathrm{H}_{5}\right), 2.77$ (d, $J=$ $\left.14.7 \mathrm{~Hz}, 3 \mathrm{H}, \mathrm{PCH}_{3}\right), 0.41(\mathrm{~s}, 9 \mathrm{H})$ und $0.36(\mathrm{~d}, J=0.5 \mathrm{~Hz}, 9 \mathrm{H})$ [ $\left.\mathrm{Si}\left(\mathrm{CH}_{3}\right)_{3}\right] .-\mathrm{IR}\left(\mathrm{v}_{\mathrm{CO}}, \mathrm{CH}_{2} \mathrm{Cl}_{2}\right): 2042 \mathrm{~cm}^{-1}$ st, 1950 sst, br.

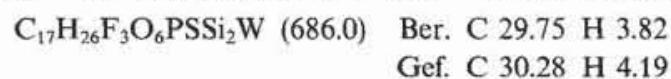

\{[Bis(trimethylsilyl) methylen] phosphan\} $/ \eta^{3}$-pentamethylcyclopentadienyl)(triphenylphosphan) nickel-trifluormethansulfonat (10c): $\mathrm{Zu}$ einer Lösung von $0.644 \mathrm{~g}(1 \mathrm{mmol}) 3 \mathrm{i}$ in $3.5 \mathrm{ml} \mathrm{CH}_{2} \mathrm{Cl}_{2}$ werden bei $-78^{\circ} \mathrm{C} 0.150 \mathrm{~g}(1 \mathrm{mmol})$ Triflatsäure gegeben. Die Lösung wird ${ }^{31} \mathrm{P}$-NMR-spektroskopisch untersucht. Versuche zur Aufarbeitung (analog 10a) gelingen in diesem Fall nicht; man erhält stets Produktgemische mit hohen Anteilen (30-50\%) unidentifizierter Zersetzungsprodukte. - ${ }^{31} \mathrm{P}-\mathrm{NMR}\left(\mathrm{CD}_{2} \mathrm{Cl}_{2}\right): \delta=271.7$ (d, $J=59.2$ $\mathrm{Hz}, \mathrm{P}=\mathrm{C}), 39.6\left(\mathrm{~d}, J=59.2 \mathrm{~Hz}, \mathrm{PPh}_{3}\right) .-{ }^{\mathrm{t}} \mathrm{H}-\mathrm{NMR}\left(\mathrm{CD}_{2} \mathrm{Cl}_{2}\right): \delta$ $=7.63(\mathrm{dd}, J=307,4 \mathrm{~Hz}, 1 \mathrm{H}, \mathrm{HP}=\mathrm{C}), 7.65(\mathrm{~m}, 6 \mathrm{H})$ und 7.52 $(\mathrm{m}, 9 \mathrm{H})\left(\mathrm{PC}_{6} \mathrm{H}_{5}\right), 1.45\left[\mathrm{dd}, J=2.4 / 4.2 \mathrm{~Hz}, 15 \mathrm{H}, \mathrm{C}_{5}\left(\mathrm{CH}_{3}\right)_{5}\right], 0.06(\mathrm{~s}$, $9 \mathrm{H})$ und $0.03(\mathrm{~s}, 9 \mathrm{H})\left[\mathrm{Si}\left(\mathrm{CH}_{3}\right)_{3}\right]$.

Tricarbonyl $\left(\eta^{5}\right.$-cyclopentadienyl $)-\mu-\{[$ phenyl(trimethylsilyl $)$ methylen] phosphanyl\}-[tetracarbonyleisen (0)] wolfram (11 a): Eine Lösung von $0.49 \mathrm{~g}(0.94 \mathrm{mmol}) 4 \mathrm{c}$ in $20 \mathrm{ml}$ Toluol wird bei $-78^{\circ} \mathrm{C}$ mit $0.341 \mathrm{~g}(0.94 \mathrm{mmol}) \mathrm{Fe}_{2}(\mathrm{CO})_{9}$ versetzt und $3 \mathrm{~h}$ gerührt. Dann wird i.Vak. bis auf $10 \mathrm{ml}$ eingedampft, mit $20 \mathrm{ml}$ Pentan versetzt und Unlösliches abgetrennt. Das Filtrat wird i.Vak. bis zur Trokkene eingedampft und 11a chromatographisch gereinigt (Säule 1 $\times 15 \mathrm{~cm}, \mathrm{Al}_{2} \mathrm{O}_{3} \mathrm{II}$, Toluol). Die rotbraune Fraktion wird aufgefan-

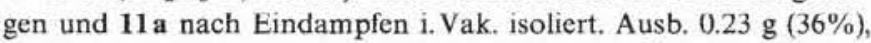
rotbraunes Pulver, Schmp. $59^{\circ} \mathrm{C}$ (Zers.) (DTA). - ${ }^{31} \mathrm{P}-\mathrm{NMR}\left(\mathrm{C}_{6} \mathrm{D}_{6}\right)$ : $\delta=316.7\left({ }^{1} J_{\mathrm{WP}}=78.4 \mathrm{~Hz}\right), 305.2\left({ }^{1} J_{\mathrm{WP}}=86.5,{ }^{2} J_{\mathrm{PCSi}}=13.4 \mathrm{~Hz}\right)$. - ${ }^{1} \mathrm{H}-\mathrm{NMR}\left(\mathrm{C}_{6} \mathrm{D}_{6}\right): \delta=7.25-6.80\left(\mathrm{~m}, 5 \mathrm{H}, \mathrm{C}_{6} \mathrm{H}_{5}\right), 4.75\left(\mathrm{~d},{ }^{3} J_{\mathrm{HCWP}}\right.$ $\left.=1.0 \mathrm{~Hz}, 5 \mathrm{H}, \mathrm{C}_{5} \mathrm{H}_{5}\right), 4.58\left(\mathrm{~d},{ }^{3} J_{\mathrm{HCWP}}=0.7 \mathrm{~Hz}, 5 \mathrm{H}, \mathrm{C}_{5} \mathrm{H}_{5}\right), 0.34[\mathrm{~s}$, $\left.9 \mathrm{H}, \mathrm{Si}\left(\mathrm{CH}_{3}\right)_{3}\right], 0.26\left[\mathrm{~s}, 9 \mathrm{H}, \mathrm{Si}\left(\mathrm{CH}_{3}\right)_{3}\right] .-{ }^{29} \mathrm{Si}-\mathrm{NMR}\left(\mathrm{C}_{6} \mathrm{D}_{6}\right): \delta=-1.2$ $\left(\mathrm{d},{ }^{2} J_{\mathrm{SiCP}}=13.2 \mathrm{~Hz}\right),-2.0\left(\mathrm{~d},{ }^{2} J_{\mathrm{SiCP}}=2.9 \mathrm{~Hz}\right) .-\mathrm{IR}\left(v_{\mathrm{CO}}\right.$, Toluol $):$ $2041 \mathrm{~cm}^{-1} \mathrm{~m}, 2010$ sst, 1961 sst, $1904 \mathrm{~m}$.

$$
\begin{array}{ll}
\mathrm{C}_{22} \mathrm{H}_{19} \mathrm{FeO}_{7} \mathrm{PSiW} \text { (694.0) } & \text { Ber. C } 38.07 \text { H } 2.76 \\
& \text { Gef. C } 37.93 \text { H } 2.82
\end{array}
$$

$\mu$-\{[Bis(trimethylsilyl) methylen]phosphanyl\}-tricarbonyl $\left(\eta^{3}-c y\right.$ clopentadienyl) [tricarbonylnickel( 0 )] wolfram (11 b): Zu einer Lösung von $1.04 \mathrm{~g}(2 \mathrm{mmol}) 3 \mathrm{c}$ in $7.5 \mathrm{ml}$ Hexan werden $0.52 \mathrm{~g} \mathrm{(3}$ mmol) Tetracarbonylnickel kondensiert. Es wird auf $0^{\circ} \mathrm{C}$ erwärmt und $1 \mathrm{~h}$ gerührt. Anschließend wird die Lösung auf $3 \mathrm{ml}$ eingeengt und das Produkt durch Abkühlen auf $-35^{\circ} \mathrm{C}$ ausgefällt. Man erhält ein rotbraunes Pulver vom Schmp. $53-59^{\circ} \mathrm{C}$ (Zers.), Ausb. $0.52 \mathrm{~g}(39 \%)$, das extrem unbeständig ist und unter Bildung von $\mathrm{Ni}(\mathrm{CO})_{4}$ und $12 \mathrm{c}$ zerfällt. - ${ }^{31} \mathrm{P}-\mathrm{NMR}\left(\mathrm{CD}_{2} \mathrm{Cl}_{2}\right): \delta=423.0\left({ }^{1} J_{\text {WP }}\right.$ $=417 \mathrm{~Hz}$ ).

$$
\begin{array}{ll}
\mathrm{C}_{18} \mathrm{H}_{23} \mathrm{NiO}_{6} \mathrm{PSi}_{2} \mathrm{~W} \text { (664.0) } & \text { Ber. C } 32.51 \text { H } 3.49 \\
& \text { Gef. C } 35.11 \text { H } 4.45
\end{array}
$$

Tricarbonyl[chlorogold(I)]( $\eta^{5}$-cyclopentadienyl)- $\mu$-\{ [ phenyl(trimethylsilyl)methylen]phosphanyl\}wolfram (11 c): Eine Lösung von $0.263 \mathrm{~g}(0.5 \mathrm{mmol}) 4 \mathrm{c}$ in $20 \mathrm{ml}$ Toluol wird bei $-78^{\circ} \mathrm{C}$ mit $0.153 \mathrm{~g}$ $(0.5 \mathrm{mmol})\left(\mathrm{Ph}_{3} \mathrm{P}\right) \mathrm{AuCl}$ versetzt. Nach Erwärmen auf Raumtemp. wird $2 \mathrm{~h}$ gerührt, dann unter Lichtausschluß das Solvens bis auf 5 $\mathrm{ml}$ eingeengt, 11 c durch Zugabe von $30 \mathrm{ml}$ Pentan ausgefällt, abgetrennt, mit $5 \mathrm{ml}$ Pentan gewaschen und i. Vak. getrocknet. Ausb. $0.25 \mathrm{~g}(68 \%)$, ockerfarbenes Pulver, Schmp. $80^{\circ} \mathrm{C}$ (Zers.). - ${ }^{31} \mathrm{P}$ NMR $\left(\mathrm{C}_{6} \mathrm{D}_{6}\right): \delta=257.9\left({ }^{1} J_{\mathrm{WP}}=117.2 \mathrm{~Hz}\right) .-{ }^{1} \mathrm{H}-\mathrm{NMR}\left(\mathrm{C}_{6} \mathrm{D}_{6}\right)$ : $\delta=7.20-6.80\left(\mathrm{~m}, 5 \mathrm{H}, \mathrm{C}_{6} \mathrm{H}_{5}\right), 4.64\left(\mathrm{~d},{ }^{3} J_{\mathrm{HCWP}}=1.0 \mathrm{~Hz}, 5 \mathrm{H}, \mathrm{C}_{5} \mathrm{H}_{5}\right)$, $0.42\left[\mathrm{~s}, 9 \mathrm{H}, \mathrm{Si}\left(\mathrm{CH}_{3}\right)_{3}\right] .-{ }^{29} \mathrm{Si}-\mathrm{NMR}\left(\mathrm{C}_{6} \mathrm{D}_{6}\right): \delta=-0.8\left({ }^{2} J_{\mathrm{SiCP}}=11.7\right.$ Hz). - IR (v co, Toluol): $2029 \mathrm{~cm}^{-1}$ sst, 1953 sst.

$$
\mathrm{C}_{18} \mathrm{H}_{19} \mathrm{AuClO}_{3} \text { PSiW (758.0) Ber. C } 28.50 \mathrm{H} 2.52 \mathrm{Cl} 4.67
$$
Gef. C 27.96 H 2.45 Cl 4.91 
$\left\{[\right.$ Bis (trimethylsilyl) methylen]phosphanyl $\}$ dicarbonyl $\left(\eta^{5}\right.$-pentamethylcyclopentadienyl)molybdän(II) (12b) und \{[Bis(trimethylsilyl) methylen] phosphanyl $\}$ dicarbonyl $\left(\eta^{5}\right.$-pentamethylcyclopentadienyl) wolfram (II) (12d)

Methode A: Lösungen von $3 \mathrm{mmol} \mathbf{3 b}$, $\mathbf{d}$ in $20 \mathrm{ml}$ Toluol werden $5-7 \mathrm{~h}$ (IR-Kontrolle) auf $70^{\circ} \mathrm{C}$ erwärmt. Nach dem Abkühlen wird das Lösungsmittel i.Vak. entfernt, und $\mathbf{1 2}$ b, $\mathbf{d}$ werden aus Pentan bei $-80^{\circ} \mathrm{C}$ kristallisiert. Ausb. 12 b: $0.43 \mathrm{~g}(30 \%)$; $12 \mathrm{~d}$ : $0.39 \mathrm{~g}(23 \%)$.

Methode B: $5 \mathrm{mmol}(\mathrm{MeCN})_{3}(\mathrm{CO})_{3} \mathrm{M}(\mathrm{M}=\mathrm{Mo}$, W) werden mit einer Lösung von $5 \mathrm{mmol} 5 \mathrm{a}$ in $20 \mathrm{ml}$ Toluol versetzt. Man erwärmt auf $65^{\circ} \mathrm{C}$ und rührt bis zum Ende der Reaktion [ ${ }^{31} \mathrm{P}-\mathrm{NMR}$-spektroskopische Reaktionskontrolle 24 h (3b), 72 h (3d)]. Anschließend wird das Lösungsmittel i. Vak. entfernt, der Rückstand in Hexan aufgenommen und Unlösliches abfiltriert. Nach mehrfacher Umkristallisation aus Hexan bei $-80^{\circ} \mathrm{C}$ erhält man $\mathbf{1 2} \mathbf{b}$, $\mathbf{d}$ als rote Kristalle. Ausb. 0.43 g 12b (18\%), 0.20 g 12d (7\%).

12 b: Schmp. $67^{\circ} \mathrm{C}$ (Zers.). - ${ }^{31} \mathrm{P}-\mathrm{NMR}\left(\mathrm{C}_{6} \mathrm{D}_{6}\right): \delta=493$ (s). ${ }^{13} \mathrm{C}-\mathrm{NMR}\left(\mathrm{C}_{6} \mathrm{D}_{6}\right): \delta=334.0(\mathrm{~d}, J=15.4 \mathrm{~Hz}, \mathrm{CO}), 132.8(\mathrm{~d}, J=$ $59.8 \mathrm{~Hz}, \mathrm{P}=\mathrm{C}), 105.2\left[\mathrm{~s}, \mathrm{C}_{5}\left(\mathrm{CH}_{3}\right)_{5}\right], 11.5\left[\mathrm{~s}, \mathrm{C}_{5}\left(\mathrm{CH}_{3}\right)_{5}\right], 2.9[\mathrm{~s}$, $\left.\mathrm{Si}\left(\mathrm{CH}_{3}\right)_{3}\right] .-{ }^{1} \mathrm{H}-\mathrm{NMR}\left(\mathrm{C}_{6} \mathrm{D}_{6}\right): \delta=1.95\left(\mathrm{~d}, J=1.6 \mathrm{~Hz}, 15 \mathrm{H}, \mathrm{CCH}_{3}\right)$, $0.40\left[\mathrm{~s}, 18 \mathrm{H}, \mathrm{Si}\left(\mathrm{CH}_{3}\right)_{3}\right]$. - MS, $m / z(\%): 478(8)\left[\mathrm{M}^{+}\right], 422(23)\left[\mathrm{M}^{+}\right.$ $-2 \mathrm{CO}], 420(26)\left[\mathrm{M}^{+}-2 \mathrm{CO}-\mathrm{H}_{2}\right], 73(100)\left[\mathrm{Me}_{3} \mathrm{Si}^{+}\right] .-\mathrm{IR}$ ( $v_{\mathrm{CO}}$, Heptan): $1961 \mathrm{~cm}^{-1}$ st, 1901 st.

$$
\begin{array}{ll}
\mathrm{C}_{19} \mathrm{H}_{33} \mathrm{MoO}_{2} \mathrm{PSi}_{2} \text { (478.1) } & \text { Ber. C } 47.69 \text { H } 6.96 \\
& \text { Gef. C } 45.83 \text { H } 6.88
\end{array}
$$

12d: Schmp. $69^{\circ} \mathrm{C}$ (Zers.). $-{ }^{31} \mathrm{P}-\mathrm{NMR}\left(\mathrm{C}_{6} \mathrm{D}_{6}\right): \delta=448.0\left({ }_{\mathrm{WP}}\right.$ $=622 \mathrm{~Hz}) .-{ }^{13} \mathrm{C}-\mathrm{NMR}\left(\mathrm{C}_{6} \mathrm{D}_{6}\right): \delta=223.6(\mathrm{~s}, \mathrm{CO}), 103.7[\mathrm{~d}, J=$ $\left.1.7 \mathrm{~Hz}, \mathrm{C}_{5}\left(\mathrm{CH}_{3}\right)_{5}\right], 11.4\left[\mathrm{~s}, \mathrm{C}_{5}\left(\mathrm{CH}_{3}\right)_{5}\right], 2.9\left[\mathrm{~d}, J=5.0 \mathrm{~Hz}, \mathrm{Si}\left(\mathrm{CH}_{3}\right)_{3}\right]$. - ${ }^{1} \mathrm{H}-\mathrm{NMR}\left(\mathrm{C}_{6} \mathrm{D}_{6}\right): \delta=2.00\left(\mathrm{~d}, J=1.5 \mathrm{~Hz}, 15 \mathrm{H}, \mathrm{CH}_{3}\right), 0.40[\mathrm{~s}$, $\left.18 \mathrm{H}, \mathrm{Si}\left(\mathrm{CH}_{3}\right)_{3}\right] .-\mathrm{MS}, m / z(\%): 564(7)\left[\mathrm{M}^{+}\right], 536(1)\left[\mathrm{M}^{+}-\mathrm{CO}\right]$, 508 (7) $\left[\mathrm{M}^{+}-2 \mathrm{CO}\right], 506$ (9) $\left[\mathrm{M}^{+}-2 \mathrm{CO}-\mathrm{H}_{2}\right], 491$ (11) [M+ - $\left.\mathrm{Me}_{3} \mathrm{Si}\right], 73$ (100) $\left[\mathrm{Me}_{3} \mathrm{Si}^{+}\right]$. - IR ( $\left.v_{\mathrm{CO}}, \mathrm{Hexan}\right): 1957 \mathrm{~cm}^{-1} \mathrm{st}$, 1894 st.

$$
\begin{array}{ll}
\mathrm{C}_{19} \mathrm{H}_{33} \mathrm{O}_{2} \mathrm{PSi}_{2} \mathrm{~W}(564.1) & \text { Ber. C } 40.43 \text { H } 5.92 \\
& \text { Gef. C } 40.54 \text { H } 5.92
\end{array}
$$

$\left\{\left[\right.\right.$ Bis(trimethylsilyl) methylen]phosphanyl \}dicarbonyl $\left(\eta^{5}-c y\right.$ clopentadienyl) wolfram (12c): Eine Lösung aus $1.04 \mathrm{~g}$ ( $2 \mathrm{mmol}) 3 \mathrm{c}$ in $5 \mathrm{ml}$ Benzol wird $22 \mathrm{~h}$ auf $70-75^{\circ} \mathrm{C}$ erwärmt. Nach Abkühlen entfernt man das Solvens i.Vak. und kristallisiert den Rückstand aus Pentan um. Man erhält $0.51 \mathrm{~g}(52 \%)$ eines roten Kristallpulvers, Schmp. $84-88^{\circ} \mathrm{C}$ (Zers.). - ${ }^{31} \mathrm{P}-\mathrm{NMR}\left(\mathrm{C}_{6} \mathrm{D}_{6}\right): \delta=445.9\left(J_{\mathrm{WP}}=\right.$ $618.2 \mathrm{~Hz}) .-{ }^{13} \mathrm{C}-\mathrm{NMR}\left(\mathrm{C}_{6} \mathrm{D}_{6}\right): \delta=218.9\left(\mathrm{~d}, J_{\mathrm{PWC}}=9.1 \mathrm{~Hz}, \mathrm{CO}\right)$, $123.9\left(\mathrm{~d}, J_{\mathrm{PC}}=47.7 \mathrm{~Hz}, \mathrm{P}=\mathrm{C}\right), 88.6\left(\mathrm{~s}, \mathrm{C}_{5} \mathrm{H}_{5}\right), 3.0\left[\mathrm{~d}, J_{\mathrm{PCSiC}}=4.7\right.$ $\left.\mathrm{Hz}, \mathrm{Si}\left(\mathrm{CH}_{3}\right)_{3}\right] .-{ }^{1} \mathrm{H}-\mathrm{NMR}\left(\mathrm{C}_{6} \mathrm{D}_{6}\right): \delta=4.79\left(\mathrm{~s}, 5 \mathrm{H}, \mathrm{C}_{5} \mathrm{H}_{5}\right), 0.32[\mathrm{~s}$, $18 \mathrm{H}, \mathrm{Si}\left(\mathrm{CH}_{3}\right)_{3}$ ]. - IR (v $\left.\mathrm{v}_{\mathrm{CO}}, \mathrm{Hexan}\right): 1952 \mathrm{~cm}^{-1}$ st, 1880 st.

$$
\begin{array}{ll}
\mathrm{C}_{14} \mathrm{H}_{23} \mathrm{O}_{2} \mathrm{PSi}_{2} \mathrm{~W}(494.0) & \text { Ber. C } 34.03 \mathrm{H} 4.69 \\
& \text { Gef. C } 33.49 \mathrm{H} 4.42
\end{array}
$$

$\left\{\left[\right.\right.$ Bis(trimethylsilyl)methylen]phosphanylthio\}tricarbonyl $\left(\eta^{5}-c y-\right.$ clopentadienyl)wolfram (14a): Eine auf $5^{\circ} \mathrm{C}$ gekühlte Lösung von $1.04 \mathrm{~g}(2.0 \mathrm{mmol}) 3 \mathrm{c}$ in $10 \mathrm{ml}$ Benzol wird mit $0.064 \mathrm{~g}(2.0 \mathrm{mmol})$ Schwefel versetzt und bis zur Auflösung des Bodenkörpers gerührt (2 h). Anschließend wird das Lösungsmittel i.Vak. entfernt und der Rückstand aus Benzol/Hexan umkristallisiert. Nach mehrfachem Waschen mit Hexan und Trocknen i.Vak. erhält man $0.99 \mathrm{~g}(90 \%)$ eines roten Kristallpulvers, Schmp. $51^{\circ} \mathrm{C}$ (Zers.). - ${ }^{31} \mathrm{P}$-NMR $\left(\mathrm{C}_{6} \mathrm{D}_{6}\right): \delta=417\left(J_{\mathrm{WP}}=14 \mathrm{~Hz}\right) .-{ }^{13} \mathrm{C}-\mathrm{NMR}\left(\mathrm{C}_{6} \mathrm{D}_{6}\right): \delta=226.6(\mathrm{~s}$, trans-CO), $214.0\left(\mathrm{~s}\right.$, cis-CO), $197.3\left(\mathrm{~d}, J_{\mathrm{PC}}=110.5 \mathrm{~Hz}, \mathrm{P}=\mathrm{C}\right), 93.1$ $\left(\mathrm{s}, \mathrm{C}_{5} \mathrm{H}_{5}\right), 2.9\left(\mathrm{~s}, \mathrm{SiCH}_{3}\right), 2.5\left(\mathrm{~d}, J_{\mathrm{PCSiC}}=13.9 \mathrm{~Hz}, \mathrm{SiCH}_{3}\right) .-{ }^{1} \mathrm{H}-$ $\operatorname{NMR}\left(\mathrm{C}_{6} \mathrm{D}_{6}\right): \delta=4.96\left(\mathrm{~s}, 5 \mathrm{H}, \mathrm{C}_{5} \mathrm{H}_{5}\right), 0.51\left(\mathrm{~s}, 9 \mathrm{H}, \mathrm{SiCH}_{3}\right), 0.33$ (d,
$\left.J_{\mathrm{PCSiCH}}=2.2 \mathrm{~Hz}, 9 \mathrm{H}, \mathrm{SiCH}_{3}\right) .-$ IR $\left(\mathrm{v}_{\mathrm{CO}}\right.$, Toluol): $2034 \mathrm{~cm}^{-1} \mathrm{st}$, 1960 st, 1952 st.

$$
\begin{array}{ll}
\mathrm{C}_{15} \mathrm{H}_{23} \mathrm{O}_{3} \mathrm{PSSi}_{2} \mathrm{~W} \text { (554.0) } & \text { Ber. C } 32.48 \mathrm{H} 4.18 \\
& \text { Gef. C } 31.12 \mathrm{H} 3.52
\end{array}
$$

\{[Bis(trimethylsilyl) methylen]phosphanylseleno\}tricarbonyl $\left(\eta^{5}\right.$ cyclopentadienyl)wolfram (14 b): Eine Lösung aus $1.07 \mathrm{~g}$ (2.1 $\mathrm{mmol})$ $3 \mathrm{c}$ in $10 \mathrm{ml} \mathrm{Benzol}$ wird mit $0.17 \mathrm{~g}(2.1 \mathrm{mmol})$ grauem Selen versetzt und $20 \mathrm{~h}$ bei Raumtemp. gerührt. Anschließend wird Unlösliches abfiltriert und der Rückstand aus Pentan umkristallisiert. Man erhält $1.04 \mathrm{~g}(82 \%)$ eines oragenroten Kristallpulvers. Schmp. $62^{\circ} \mathrm{C}$ (Zers.). $-{ }^{31} \mathrm{P}-\mathrm{NMR}\left(\mathrm{C}_{6} \mathrm{D}_{6}\right): \delta=431.8\left(J_{\mathrm{PSeW}}=11.9, J_{\mathrm{PSe}}=336.9\right.$ $\mathrm{Hz}) .-{ }^{13} \mathrm{C}-\mathrm{NMR}\left(\mathrm{C}_{6} \mathrm{D}_{6}\right): \delta=224.8$ (s, trans-CO), 213.3 (d, $J_{\mathrm{PSeWC}}$ $=6.7 \mathrm{~Hz}$, cis-CO), $198.3\left(\mathrm{~d}, J_{\mathrm{PC}}=111.6 \mathrm{~Hz}, \mathrm{P}=\mathrm{C}\right), 92.3\left(\mathrm{~s}, \mathrm{C}_{\mathrm{s}} \mathrm{H}_{5}\right)$, $1.36\left(\mathrm{~s}, \mathrm{SiCH}_{3}\right) .-{ }^{1} \mathrm{H}-\mathrm{NMR}\left(\mathrm{C}_{6} \mathrm{D}_{6}\right): \delta=4.76\left(\mathrm{~s}, 5 \mathrm{H}, \mathrm{C}_{5} \mathrm{H}_{5}\right), 0.60(\mathrm{~s}$, $\left.9 \mathrm{H}, \mathrm{SiCH}_{3}\right), 0.33$ (d, $\left.J_{\mathrm{PCSiCH}}=2.7 \mathrm{~Hz}, 9 \mathrm{H}, \mathrm{SiCH}_{3}\right) .-$ IR $\left(v_{\mathrm{CO}}\right.$, Pentan): $2025 \mathrm{~cm}^{-1}$ vs, 1954 vs, 1946 vs.

$$
\begin{array}{ll}
\mathrm{C}_{15} \mathrm{H}_{23} \mathrm{O}_{3} \mathrm{PSeSi}_{2} \mathrm{~W} \text { (601.3) } & \text { Ber. C } 29.96 \text { H } 3.86 \\
& \text { Gef. C } 29.85 \text { H } 3.81
\end{array}
$$

Tricarbonyl ( $\eta^{5}$-cyclopentadienyl) \{[phenyl (trimethylsilyl) methylen]phosphanylseleno \}wolfram (14c): Eine Lösung von $0.30 \mathrm{~g}(0.58$ $\mathrm{mmol}) 4 \mathrm{c}$ in $20 \mathrm{ml}$ Toluol wird mit $0.045 \mathrm{~g}(0.58 \mathrm{mmol})$ grauem Selen versetzt und $2 \mathrm{~h}$ gerührt. Anschließend wird Unlösliches abgetrennt und das Lösungsmittel i.Vak. entfernt. Der Rückstand

\begin{tabular}{|c|c|c|c|c|}
\hline & $\mathrm{x}$ & $\mathrm{y}$ & $\mathrm{z}$ & $\mathrm{U}(\mathrm{eq})$ \\
\hline $\mathrm{Fe}(1)$ & $659(1)$ & $3383(1)$ & $722(1)$ & $59(1)$ \\
\hline$c(1)$ & $-615(5)$ & $4430(4)$ & $857(2)$ & $77(2)$ \\
\hline $0(1)$ & $-1463(4)$ & $5111(3)$ & $929(2)$ & $110(2)$ \\
\hline$c(2)$ & $-508(4)$ & $2323(4)$ & $956(2)$ & $72(2)$ \\
\hline$O(2)$ & $-1244(4)$ & $1639(3)$ & $1112(2)$ & $104(1)$ \\
\hline$c(3)$ & $1923(5)$ & $4428(4)$ & $534(2)$ & $77(2)$ \\
\hline $0(3)$ & $2709(4)$ & $5111(3)$ & $417(2)$ & $122(2)$ \\
\hline$C(4)$ & $498(5)$ & $2809(4)$ & $28(2)$ & $87(2)$ \\
\hline $0(4)$ & $336(5)$ & $2480(3)$ & $-414(1)$ & $134(2)$ \\
\hline$P(1)$ & $2330(1)$ & $2733(1)$ & $1363(1)$ & $53(1)$ \\
\hline$c(5)$ & $2348(4)$ & $2947(3)$ & $2052(1)$ & $51(1)$ \\
\hline Si(1) & $1032(1)$ & $3922(1)$ & $2382(1)$ & $66(1)$ \\
\hline$c(6)$ & $1496(6)$ & $3899(5)$ & $3155(2)$ & $135(3)$ \\
\hline$c(7)$ & $-907(4)$ & $3473(3)$ & $2257(2)$ & $77(2)$ \\
\hline$c(8)$ & $1270(5)$ & $5366(3)$ & $2132(2)$ & $111(2)$ \\
\hline$C(9)$ & $3411(4)$ & $2420(3)$ & $2482(1)$ & $50(1)$ \\
\hline$c(10)$ & $3057(5)$ & $1427(3)$ & $2735(2)$ & $71(2)$ \\
\hline$C(11)$ & $3965(6)$ & $964(5)$ & $3150(2)$ & $95(2)$ \\
\hline$C(12)$ & $5236(6)$ & $1462(5)$ & $3319(2)$ & $98(2)$ \\
\hline$C(13)$ & $5629(5)$ & $2453(5)$ & $3082(2)$ & $90(2)$ \\
\hline$C(14)$ & $4701(4)$ & $2942(3)$ & $2662(2)$ & $67(1)$ \\
\hline$C(15)$ & $3762(4)$ & $1767(3)$ & 1121 (1) & $56(1)$ \\
\hline$C(16)$ & $2932(4)$ & $851(3)$ & $809(2)$ & $64(1)$ \\
\hline$c(17)$ & $3135(5)$ & $947(4)$ & $269(2)$ & $76(2)$ \\
\hline$C(18)$ & $4046(5)$ & $1886(4)$ & $173(2)$ & $78(2)$ \\
\hline$C(19)$ & $4433(4)$ & $2391(3)$ & $656(2)$ & $67(1)$ \\
\hline$C(20)$ & $4939(5)$ & $1297(4)$ & $1550(2)$ & $98(2)$ \\
\hline$C(21)$ & $2084(6)$ & $-11(4)$ & $1100(2)$ & $113(2)$ \\
\hline$C(22)$ & $2528(6)$ & $160(5)$ & $-195(2)$ & $137(3)$ \\
\hline$c(23)$ & $4487(7)$ & $2222(6)$ & $-401(2)$ & $149(3)$ \\
\hline$C(24)$ & $5452(5)$ & $3355(4)$ & $773(2)$ & $120(3)$ \\
\hline
\end{tabular}
wird mit einem Gemisch aus $3 \mathrm{ml}$ Toluol und $20 \mathrm{ml}$ Pentan $12 \mathrm{~h}$ behandelt, danach abfiltriert, zurückbleibendes $14 \mathrm{e}$ mit $5 \mathrm{ml}$ Pentan gewaschen und i.Vak. getrocknet. Ausb. $0.16 \mathrm{~g} \mathrm{(47 \% )}$ orangerotes Pulver, Schmp. $55^{\circ} \mathrm{C}$ (Zers.). - ${ }^{31} \mathrm{P}-\mathrm{NMR}\left(\mathrm{C}_{3} \mathrm{D}_{6} \mathrm{O}\right): \delta=342.9\left({ }^{2} J_{\mathrm{PCSi}}\right.$ $\left.=44.0,{ }^{1} J_{\mathrm{PSeW}}=12.5,{ }^{1} J_{\mathrm{PSe}}=326.1 \mathrm{~Hz}\right) .-{ }^{1} \mathrm{H}-\mathrm{NMR}\left(\mathrm{C}_{6} \mathrm{D}_{6}\right): \delta=$

Tab. 3. Atomkoordinaten $\left(-10^{4}\right)$ und äquivalente isotrope thermische Parameter $\left[\mathrm{pm}^{2} \cdot 10^{-1}\right]$ von $6 \mathrm{a}$. Äquivalente isotrope $U$ berechnet als ein Drittel der Spur des $U_{i j}$-Tensors 
$7.31-6.98\left(\mathrm{~m}, 5 \mathrm{H}, \mathrm{C}_{6} \mathrm{H}_{5}\right), 4.53\left(\mathrm{~s}, 5 \mathrm{H}, \mathrm{C}_{5} \mathrm{H}_{5}\right), 0.21\left[\mathrm{~d},{ }^{4} J_{\mathrm{HCSiCP}}=1.2\right.$ $\left.\mathrm{Hz}, 9 \mathrm{H}, \mathrm{Si}\left(\mathrm{CH}_{3}\right)_{3}\right] .-{ }^{29} \mathrm{Si}-\mathrm{NMR}\left(\mathrm{C}_{3} \mathrm{D}_{6} \mathrm{O}\right): \delta=-3.8\left(\mathrm{~d},{ }^{2} J_{\mathrm{SiCP}}=\right.$ $44.0 \mathrm{~Hz}$ ). - IR ( $v_{\mathrm{CO}}$, Methylcyclohexan): $2024 \mathrm{~cm}^{-1}$ vs, $1952 \mathrm{vs,}$ 1944 vs.

$$
\mathrm{C}_{18} \mathrm{H}_{19} \mathrm{O}_{3} \mathrm{PSeSiW}(606.0) \quad \text { Ber. C } 35.72 \mathrm{H} 3.16
$$$$
\text { Gef. C } 35.14 \text { H } 3.00
$$

Tricarbonyl ( $\eta^{5}$-cyclopentadienyl) [3-phenyl-3-(trimethylsilyl)-2thioxo-1,2 $\lambda^{5}$-thiaphosphiran-2-yl]wolfram (15): Eine Lösung von $0.15 \mathrm{~g}(0.29 \mathrm{mmol}) 4 \mathrm{c}$ in $10 \mathrm{ml}$ Benzol wird bei Raumtemp. mit $0.05 \mathrm{~g}$ ( $1.56 \mathrm{mmol})$ elementarem Schwefel versetzt. Nach $4 \mathrm{~h}$ wird das Solvens i.Vak. entfernt, der Rückstand in $10 \mathrm{ml}$ Pentan aufgenommen und 15 bei $-78^{\circ} \mathrm{C}$ ausgefroren. Ausb. $0.10 \mathrm{~g}\left(65^{\circ}\right)$, braunes Pulver, Schmp. $130^{\circ} \mathrm{C}$ (Zers.). - ${ }^{31} \mathrm{P}-\mathrm{NMR}\left(\mathrm{C}_{3} \mathrm{D}_{6} \mathrm{O}\right): \delta=$ $-17.7\left({ }^{1} J_{\mathrm{WP}}=219.8 \mathrm{~Hz}\right) .-{ }^{1} \mathrm{H}-\mathrm{NMR}\left(\mathrm{C}_{3} \mathrm{D}_{6} \mathrm{O}\right): \delta=7.60-7.10$ $\left(\mathrm{m}, 5 \mathrm{H}, \mathrm{C}_{6} \mathrm{H}_{5}\right), 5.82\left(\mathrm{~d},{ }^{3} J_{\mathrm{HCWP}}=0.5 \mathrm{~Hz}, 5 \mathrm{H}, \mathrm{C}_{5} \mathrm{H}_{5}\right), 0.17[\mathrm{~s}, 9 \mathrm{H}$, $\left.\mathrm{Si}\left(\mathrm{CH}_{3}\right)_{3}\right] .-{ }^{29} \mathrm{Si}-\mathrm{NMR}\left(\mathrm{C}_{6} \mathrm{D}_{6}\right): \delta=9.3$. - IR $\left(v_{\mathrm{CO}}\right.$, Methylcyclohexan): $2037 \mathrm{~cm}^{-1}$ s, 1968 sst, 1955 sst.

$$
\begin{array}{ll}
\mathrm{C}_{18} \mathrm{H}_{19} \mathrm{O}_{3} \mathrm{PS}_{2} \mathrm{SiW} \text { (590.0) } & \text { Ber. C } 36.62 \text { H } 3.24 \mathrm{~S} 10.86 \\
& \text { Gef. C } 36.40 \text { H } 3.38 \mathrm{~S} 9.26
\end{array}
$$

Röntgenstrukturanalyse von $\mathbf{6} \mathbf{b}^{[9]}$ : Kristalldimensionen $0.5 \times 0.6$ $\times 0.8 \mathrm{~mm}$; orangefarbene Kristalle; $\mathrm{C}_{24} \mathrm{H}_{29} \mathrm{FeO}_{4} \mathrm{PSi}, M_{\mathrm{r}}=496.4 \mathrm{~g}$ $\mathrm{mol}^{-1}$; monoklin; Raumgruppe $P 2_{1} / c$ (Nr. 14); $a=9.208(2), b=$ 11.999(2), $c=23.911(6) \AA, \beta=94.50(2)^{\circ} ; V=2.634(1) \mathrm{nm}^{3} ; Z=$ $4, d_{\text {ber. }}=1.25 \mathrm{~g} \mathrm{~cm}^{-3} ; \mu\left(\mathrm{Mo}-K_{\alpha}\right)=0.70 \mathrm{~mm}^{-1}, F(000)=1040$. Mit einem Nicolet-R3m-Vierkreisdiffraktometer (Graphitmonochromator, Mo- $K_{\alpha}$-Strahlung, $\lambda=0.71073 \AA$ ) wurden 4553 symmetrieunabhängige Reflexe $\left(2 \Theta_{\max }=50^{\circ}, h:-10 \rightarrow 10, k: 0 \rightarrow 14\right.$, l: $0 \rightarrow 28 ; \omega$-Scans, Scanbreite $\left.1.20^{\circ}\right)$ bei Raumtemp. gemessen. 3497 Reflexe mit $|F|>4 \sigma(F)$ wurden zur Strukturlösung (Direkte Methoden) und -verfeinerung (280 Parameter) verwendet. Die Nicht-Wasserstoffatome wurden anisotrop, die Wasserstoffatome mit einem Reiter-Modell verfeinert. Die Verfeinerung konvergierte mit $R=0.051\left[R_{w}=0.051, w^{-1}=\sigma^{2}(F)+0.0001 F^{2}\right]$, Restelektronendichte $\left(0.29 \mathrm{e} / \AA^{3}\right)$. Strukturlösung und -verfeinerung wurden mit dem Programm SHELXTL-Plus durchgeführt (Atomkoordinaten s. Tab. 3).

* Herrn Professor Heinrich Nöth zum 65. Geburtstag gewidmet. [1] [1a] R. Appel in Multiple Bonds and Low Coordination in Phosphorus Chemistry (Ed.: M. Regitz, O. J. Scherer), Thieme Verlag,
Stuttgart, 1990, S. $191-195 .-{ }^{\left[{ }^{b b}\right]}$ J. F. Nixon, Chem. Rev. 1988, $88,1327-1362$

${ }^{[2]}$ L. Weber, K. Reizig, R. Boese, Organomet. 1985, 4, 2097-2101.

${ }^{[3]}$ A. H. Cowley, N. C. Norman, S. Quashic, J. Am. Chem. Soc. 1984, 106, 5007-5008.

${ }^{[4]}$ H. J. Metternich, E. Niecke, J. F. Nixon, J. Chem. Soc., Chem. Commun. 1992, 232-233.

[ร] [5a] W. Malisch, E. Groß, K. Jörg, M. Schmeußer, A. Meyer, R. Baumann, Phosphorus Sulfur 1986, 26, 25-26. - ${ }^{[5 b]}$ H. H. Karsch, H.-U. Reisacher, K. Jörg, W. Malisch, B. Huber, G. Müller, New J. Chem. 1989, 13, 319-327. - ${ }^{[S c]}$ U.-A. Hirth, H. Käb, Th. A. Bright, U. Weis, W. Malisch in Selective Reactions of Metal-Activated Molecules (Eds.: H. Werner, A. G. Griesbeck, W. Adam, G. Bringmann, W. Kiefer), Vieweg-Verlag, Braunschweig, 1992, S. 187-190.

${ }^{[6]}$ D. Gudat, E. Niecke, W. Malisch, U. Hofmockel, S. Quashie, A. H. Cowley, A. M. Arif, B. Krebs, M. Dartmann, J. Chem. Soc., Chem. Commun. 1985, 1687-1689.

[7] [7a] D. Gudat, E. Niecke, B. Krebs, M. Dartmann, Chimia 1985, 39, 277-279. - ${ }^{[7 b]}$ D. Gudat, E. Niecke, J. Chem. Soc., Chem. Commun. 1987, 10-11. - ${ }^{[7 c]}$ D. Gudat, M. F. Meidine, J. F. Nixon, E. Niecke, J. Chem. Soc., Chem. Commun. 1989 , $1206-1208$.

${ }^{[8]}$ D. Gudat, E. Niecke, A. M. Arif, S. Quashie, A. H. Cowley, Organomet. 1986, 5, 593-594.

${ }^{[9]}$ Weitere Einzelheiten zur Kristallstrukturanalyse können beim Fachinformationszentrum Karlsruhe, Gesellschaft für wissenschaftlich-technische Information $\mathrm{mbH}, \mathrm{D}-7514$ EggensteinLeopoldshafen 2, unter Angabe der Hinterlegungsnummer CSD-56844, der Autorennamen und des Zeitschriftenzitats angefordert werden.

${ }^{110}$ R. R. Ford, L. Bei-Li, R. H. Neilson, R. J. Thoma, Inorg. Chem. 1985, 24, 1993.

(111) $\mathrm{K}$. Karaghiosoff in Multiple Bonds and Low Coordination in Phosphorus Chemistry (Ed.: M. Regitz, O. J. Scherer), Thieme Verlag, Stuttgart, 1990, S. 463-471.

${ }^{[12]}$ L. Weber, K. Reizig, R. Boese, M. Polk, Angew. Chem. 1985, 97, 583; Angew. Chem. Int. Ed. Engl. 1985, 24, 604.

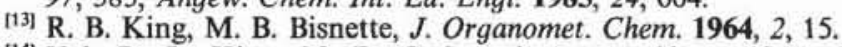

${ }^{[14]}$ Vgl. R. B. King, N. D. Sadavani, Inorg. Chem. 1985, 24, $3136-3139$.

${ }^{115]}$ W. Malisch, R. Maisch, I. J. Colquhoun, W. McFarlane, J. Organomet. Chem. 1981, 220, C1; W. Angerer, W. S. Sheldrick, W. Malisch, Chem. Ber. 1985, 118, 1261.

${ }^{[16]}$ Th. A. v. d. Knaap, M. Vos, F. Bickelhaupt, J. Organomet. Chem. 1983, 244, 363; M. Yoshifuji, K. Shibayana, N. Inamoto, $N$. Chem. Lett. 1984, 603.

${ }^{[17]}$ S. O. Grimm, D. A. Wheatland, W. McFarlane, J. Am. Chem. Soc., $1967,89,5573$

${ }^{[18]}$ W. W. Schoeller, E. Niecke, J. Chem. Soc., Chem. Commun. 1982, 569.

[391/92] 